\title{
The Molpro quantum chemistry package
}

Cite as: J. Chem. Phys. 152, 144107 (2020); https://doi.org/10.1063/5.0005081

Submitted: 19 February 2020 . Accepted: 17 March 2020 . Published Online: 13 April 2020

Hans-Joachim Werner (D), Peter J. Knowles (D), Frederick R. Manby (D), Joshua A. Black (D), Klaus Doll (D), Andreas Heßelmann (D), Daniel Kats (D), Andreas Köhn (D), Tatiana Korona (D), David A. Kreplin (D), Qianli Ma (D), Thomas F. Miller (D), Alexander Mitrushchenkov (D), Kirk A. Peterson (D), lakov Polyak (D), Guntram Rauhut (D), and Marat Sibaev (D)

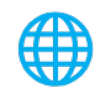

\section{Lock-in Amplifiers up to $600 \mathrm{MHz}$}
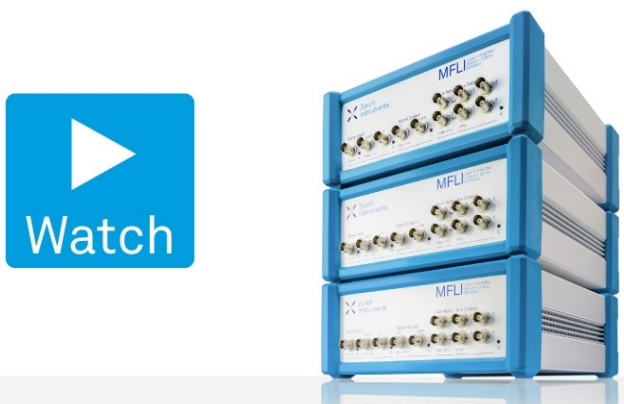


\title{
The Molpro quantum chemistry package
}

\author{
Cite as: J. Chem. Phys. 152, 144107 (2020); doi: 10.1063/5.0005081 \\ Submitted: 19 February 2020 - Accepted: 17 March 2020 • \\ Published Online: 13 April 2020
}

\begin{tabular}{|c|}
\hline 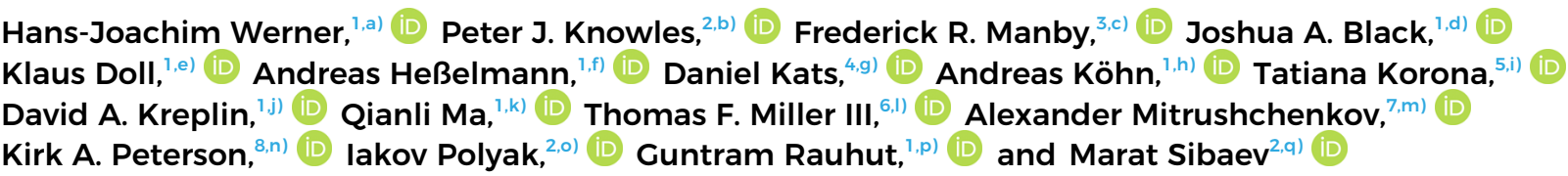 \\
\hline
\end{tabular}

\begin{abstract}
AFFILIATIONS
${ }^{1}$ Institut für Theoretische Chemie, Universität Stuttgart, Pfaffenwaldring 55, 70569 Stuttgart, Germany

${ }^{2}$ School of Chemistry, Cardiff University, Main Building, Park Place, Cardiff CF10 3AT, United Kingdom

${ }^{3}$ School of Chemistry, University of Bristol, Cantock's Close, Bristol BS8 1TS, United Kingdom

${ }^{4}$ Max-Planck Institute for Solid State Research, Heisenbergstraße 1, 70569 Stuttgart, Germany

${ }^{5}$ Faculty of Chemistry, University of Warsaw, L. Pasteura 1 St., 02-093 Warsaw, Poland

${ }^{6}$ Division of Chemistry and Chemical Engineering, California Institute of Technology, Pasadena, California 91125, USA

${ }^{7}$ MSME, Université Gustave Eiffel, UPEC, CNRS, F-77454 Marne-la-Vallée, France

${ }^{8}$ Department of Chemistry, Washington State University, Pullman, Washington 99164-4630, USA
\end{abstract}

Note: This article is part of the JCP Special Topic on Electronic Structure Software.

a) Author to whom correspondence should be addressed: werner@theochem.uni-stuttgart.de

b) Electronic mail: KnowlesPJ@Cardiff.ac.uk

${ }^{c)}$ Electronic mail: fred.manby@bristol.ac.uk

d) Electronic mail: black@theochem.uni-stuttgart.de

e) Electronic mail: doll@theochem.uni-stuttgart.de

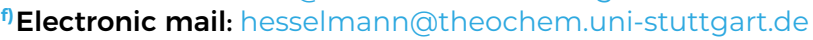

g) Electronic mail: d.kats@fkf.mpg.de

h)Electronic mail: koehn@theochem.uni-stuttgart.de

${ }^{i)}$ Electronic mail: tania@chem.uw.edu.pl

j) Electronic mail: kreplin@theochem.uni-stuttgart.de

${ }^{k)}$ Electronic mail: qianli@theochem.uni-stuttgart.de

I) Electronic mail: tfm@caltech.edu

m) Electronic mail: Alexander.Mitrushchenkov@univ-eiffel.fr

n) Electronic mail: kipeters@wsu.edu

${ }^{\circ}$ Electronic mail: Polyakl@Cardiff.ac.uk

p) Electronic mail: rauhut@theochem.uni-stuttgart.de

a) Electronic mail: SibaevM@Cardiff.ac.uk

\begin{abstract}
Molpro is a general purpose quantum chemistry software package with a long development history. It was originally focused on accurate wavefunction calculations for small molecules but now has many additional distinctive capabilities that include, inter alia, local correlation approximations combined with explicit correlation, highly efficient implementations of single-reference correlation methods, robust and efficient multireference methods for large molecules, projection embedding, and anharmonic vibrational spectra. In addition to conventional input-file specification of calculations, Molpro calculations can now be specified and analyzed via a new graphical user interface and through a Python framework.
\end{abstract}

Published under license by AIP Publishing. https://doi.org/10.1063/5.0005081 


\section{INTRODUCTION}

Molpro is a general purpose quantum chemistry software package with a development history of over 50 years. It was started in the late 1960s by the pioneering work of Meyer and Pulay: Meyer developed the pseudo-natural configuration interaction $(\mathrm{PNO}-\mathrm{CI})^{1}$ and coupled-electron pair (PNO-CEPA) ${ }^{2}$ methods and Pulay developed the first Hartree-Fock analytical energy gradient program. ${ }^{3}$ These methods have been used in the 1970s for numerous calculations of molecular properties of small molecules with unprecedented accuracy. ${ }^{4-9}$ Since then, the main focus of Molpro has been on high accuracy treatments of electron correlation. Perhaps, most widely used and distinctive are the internally contracted multireference configuration interaction (IC-MRCI) methods $^{10-14}$ in Molpro, which allowed for the first time to compute global potential energy surfaces (PES) for electronic ground and excited states (ESs) with unmatched precision. In many cases, these calculations also included non-adiabatic and spin-orbit couplings. ${ }^{15}$ The PES were used in many studies of molecular spectroscopy, as well as in quasi-classical and quantum mechanical treatments of inelastic and reactive collision dynamics. Molpro is also well known for its highly efficient coupled cluster modules with single, double, and triple excitations $[\operatorname{CCSD}(\mathrm{T})]$. Through the inclusion of explicitly correlated (F12) terms in the MP2F12 $2^{16,17}$ (explicitly correlated second-order Møller-Plesset perturbation theory), $\operatorname{CCSD}(\mathrm{T})-\mathrm{F} 12,{ }^{18-20} \mathrm{CASPT} 2-\mathrm{F} 12^{21}$ (explicitly correlated second-order complete active space perturbation theory), and MRCI-F12 $2^{22-24}$ programs, the basis set limits of the corresponding methods can be closely approached using triple- $\zeta$ basis sets. ${ }^{19,25-30}$ Typically, this reduces the basis set incompleteness errors of relative energies and molecular properties by at least one order of magnitude at rather little additional cost. Molpro also contains an efficient implementation of Kohn-Sham (KS) density functional theory (DFT) that supports standard hybrid functionals, as well as extensions including dispersion corrections, range-corrected wavefunction hybrids, and exact-exchange functionals.

The traditional functionality of Molpro has been summarized in an earlier review article ${ }^{31}$ and will, therefore, be outlined only briefly. The main focus of this paper is on new methods, and the current authors are those who contributed significantly to these developments. The full list of contributors is given in Ref. 32 . We describe improvements of coupled cluster methods [distinguishable clus$\operatorname{ter}^{33-37}$ and quasi-variational coupled-cluster (QVCC) ${ }^{38-43}$ ], as well as recent developments of accurate electron correlation treatments for large molecules. This includes highly accurate explicitly correlated local coupled cluster methods based on pair natural orbitals [PNO-LCCSD(T)-F12] ${ }^{44-58}$ and embedding methods. ${ }^{59}$ In the multireference area, a new multiconfiguration (complete active space) self-consistent field (MCSCF/CASSCF) program has been developed, ${ }^{60,61}$ which allows efficient optimizations of wavefunctions for large systems, such as transition metal clusters. The CASSCF orbitals can be used in subsequent single-state ${ }^{62}$ or multi-state (MS) ${ }^{63} \mathrm{PNO}$ CASPT2 calculations to include dynamical correlation effects in large systems.

The paper is organized as follows: in Secs. II and III, we describe the many wavefunction and density functional methods, respectively, which are available in Molpro. Section IV B presents projection-based wavefunction-in-DFT embedding methods, which allow combination of both worlds. Section V summarizes the treatment of scalar-relativistic and spin-orbit effects, and Sec. VI describes methods to compute molecular properties and molecular spectra. Section VII gives an overview of methods to compute and analyze intermolecular interaction energies, either with supermolecular approaches or using symmetry adapted perturbation theory (SAPT). Finally, in Sec. VIII, we describe a new graphical user interface (GUI) and further interfaces, which can be used for connecting Molpro with other programs or for postprocessing molecular orbitals and molecular properties.

\section{WAVEFUNCTION METHODS}

\section{A. Canonical single reference methods}

\section{Hartree-Fock and Kohn-Sham}

Closed-shell and open-shell Hartree-Fock programs are available with and without spin-restriction (RHF and UHF) and with or without a Kohn-Sham (KS) density functional. The programs can run in conventional mode using integrals stored on disk or in integral-direct mode. For large molecular systems, we recommend using the density fitting (DF) implementation, which is most efficient. These programs have been described in Ref. 31, and this is not repeated here. More recently, a new parallel version with local approximations has been developed, ${ }^{64}$ which can considerably speed up calculations for large molecules. Furthermore, a configuration averaged Hartree-Fock (CA-HF) program ${ }^{65,66}$ has been added, which is available with and without local approximations. This is equivalent to state-averaged CASSCF that includes all possible states, of any overall spin, within the active space. The method is useful for the calculation of crystal field splittings in transition metal and lanthanide complexes. The latest version ${ }^{66}$ is also applicable to polynuclear complexes. A new density fitting module for computing the Coulomb and exchange parts of the Fock matrices with much improved parallelization has also been provided. This program can be used on multi-node computer systems. An example demonstrating the efficiency and speedups is shown in Fig. 1. We note that it is sometimes difficult to achieve convergence in ROHF calculations for large open-shell transition metal complexes. In such cases, we recommend the use of our new MCSCF program (cf. Sec. II B 1), which converges more rapidly and robustly and is only slightly more expensive per iteration.

\section{MP2, coupled-cluster}

Molpro includes highly efficient implementations of all important single reference (SR) methods for closed-shell and openshell electron correlation treatments. These range from MP2 to $\operatorname{CCSD}(\mathrm{T})$, with variants such as Brueckner coupled cluster [BCCD and $\mathrm{BCCD}(\mathrm{T})],{ }^{68}$ quadratic configuration interaction [QCISD(T)], distinguishable cluster either with orbital optimization (ODCD and BDCD) or with single and double excitations (DCSD), ${ }^{33-35,37}$ and quasi-variational coupled-cluster [QVCCD(T).$^{38-43}$ The latter two methods extend the application area of single-reference coupledcluster theory to situations where multireference effects become important (see Sec. II A 3 for more details). Excited states can 


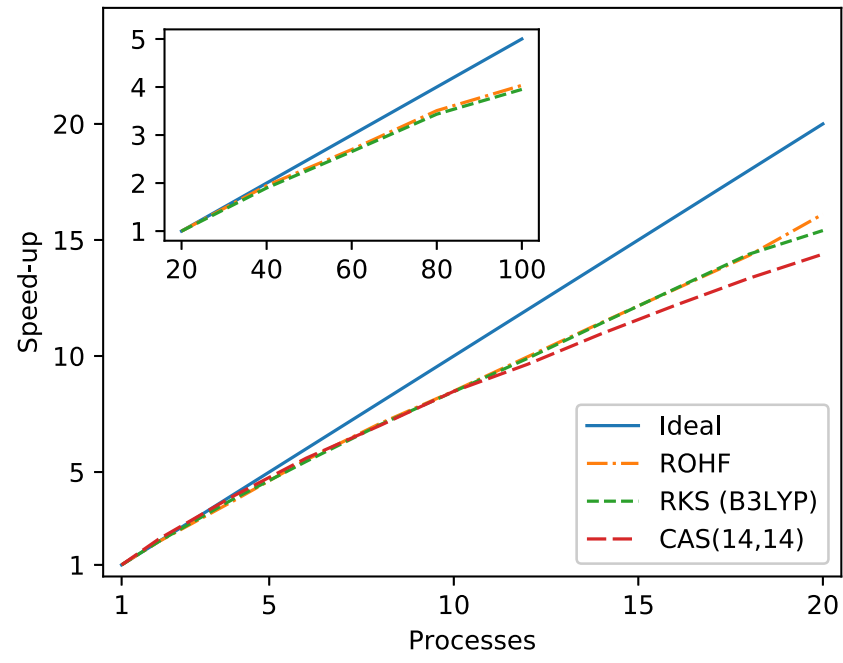

FIG. 1. Parallel speed-ups for spin-restricted Hartree-Fock and hybrid KohnSham (B3LYP) calculation of the ${ }^{7} \mathrm{~A}$ state of $\mathrm{C}_{2} \mathrm{ON}_{4} \mathrm{C}_{70} \mathrm{H}_{106}{ }^{67}$ with the def2-tzvp basis. Additionally shown is the parallel speed-up for the CASSCF calculation of the ${ }^{1} \mathrm{~A}$ state. The main figure shows parallel speed-up relative to a single MPI process on a single 20-core node; the inset shows the speed-up on multiple nodes relative to a single node computation, with 20 processes used on each node.

be treated using $\mathrm{CC} 2^{69-73}$ and equation-of-motion (EOM)-CCSD ${ }^{74}$ methods. Spin-component scaling approximations ${ }^{75}$ are available for MP2 and DCSD. ${ }^{37}$ The open-shell methods (except spinunrestricted MP2, UMP2) are based on ROHF orbitals, and partially spin restricted $[\mathrm{RCCSD}(\mathrm{T})]^{76}$ as well as unrestricted coupled-cluster implementations [ROHF-UCCSD(T)] are available. Explicitly correlated (F12) variants ${ }^{18-20,35,77}$ are implemented for all mentioned ground-state methods [except QVCCD $(\mathrm{T})$ ]. As already mentioned, the F12 terms greatly reduce the basis set incompleteness errors and are, therefore, highly recommended. For most of the methods, also analytical energy gradients are implemented, as described in Sec. VI A.

Traditionally, the MP2 and coupled-cluster methods use twoelectron integrals stored on disk. Integral direct implementations, in which the integrals are computed on the fly whenever needed, are available for QCISD and CCSD. ${ }^{78}$ Alternatively, density fitting (DF) can be used, and DF-MP2 and closed-shell DF-CCSD(T)F12 methods are also implemented in Molpro. While density fitting greatly improves the efficiency of MP2, this is much less the case for canonical coupled-cluster methods. Therefore, DF is normally not recommended for conventional CCSD and its variants. However, highly efficient and accurate closed-shell and open-shell local CCSD(T) methods for large molecules are also available in Molpro, cf. Sec. II D 3. These methods are entirely based on density fitting approximations and are recommended for calculations on molecules that are too large to be treated by conventional $\operatorname{CCSD}(\mathrm{T})$.

\section{Coupled-cluster methods for strong electron correlation}

Although multireference methods are a preferred route to describing strong electron correlation, the difficulty in controlling both their cost scaling and accuracy for large systems means that single-reference methods are attractive whenever they can be used. However, it is well understood that standard truncated coupledcluster methods, such as CCSD and CCSD(T), result in significant errors when used to describe chemical systems with strong nondynamic correlation. ${ }^{79,80}$ A number of alternative single reference (SR) methods that are able to model inherently MR systems have emerged in recent years. ${ }^{33,38,81-83}$ These include the quasi-variational coupled-cluster doubles (QVCCD) and the distinguishable cluster doubles (DCD) approaches. ${ }^{33-43}$ In essence, both of these methods attempt to improve upon CCD, either via the addition of higher order terms, as in QVCCD, or in the subtraction of specific quadratic terms, in the case of DCD. As a result, these methods can produce remarkable accuracy along dissociation paths, even though they are based on a single determinant reference.

QVCCD begins with an energy expression that takes the form of the CEPA(0), or linearized CCD, energy. However, the cluster amplitudes used in the energy expression are expressed as a linear combination of the true amplitudes that by construction yields an energy that is exact for two electrons, is extensive, and agrees with the series expansion of variational coupled-cluster doubles (VCCD) up to third-order in $\hat{T}$. The transformation matrices that satisfy these constraints are then defined as inverses and inverse square roots of reduced density matrices (RDMs) obtained from the CI wavefunction constructed from $\hat{T}$. The use of matrix powers to achieve this leads to a closed-form expression for the energy and amplitude residual equations, introducing the implicit inclusion of an infinite sub-set of higher order terms based upon the third-order VCCD terms.

The further inclusion of the one- and three-body correlations can be obtained to produce a quantitatively correct QVCCD $(\mathrm{T})$ method, which approaches chemical accuracy. The effects of the singles amplitudes are included via orbital optimization or the use of Brueckner orbitals. Connected triple excitation effects can be effectively approximated by using the standard perturbative $(\mathrm{T})$ correction. However, this has been shown to overshoot for strongly correlated regimes, ${ }^{41}$ and instead, a simple modification, effectively a renormalization of the $(\mathrm{T})$ denominator, should be used to produce accurate potential energy surfaces. ${ }^{4}$

QVCCD is now implemented in Molpro within the Integrated Tensor Framework (ITF, cf. Sec. II B 2) with similar computational scaling to CCSD but with a slightly larger prefactor because of the need to perform the extra linear algebra required in the QVCCD equations. This implementation has led to a significant speed-up compared to the older implementation, meaning that this method can be routinely used in applications.

DCD amplitude equations can be obtained from the CCD equations by removing some inter-cluster exchange diagrams ${ }^{33}$ or by accounting for all possible electron pair interactions with a RPA-screened Coulomb potential. ${ }^{36}$ The orbital relaxation can be taken into account using Brueckner orbitals (BDCD), orbitaloptimization (ODCD), or single-excitation similarity transformation (DCSD), resulting in a size-extensive two-electron-exact method. $^{3}$

The distinguishable cluster methods work remarkably well for strongly and weakly correlated systems. ${ }^{33,35,37}$ They outperform the coupled-cluster doubles methods in virtually all situations and are even on a par with $\operatorname{CCSD}(\mathrm{T})$ for the accuracy of optimized molecular 
geometries. ${ }^{35}$ Strongly correlated molecular and model systems are treated qualitatively correct. In particular, Hubbard-type one-, two-, or three-dimensional equidistant hydrogen lattices are dissociated to the exact limit.

DCSD has been combined with the explicit correlation methods (DCSD-F12) and is available as a closed-shell (together with BDCD and ODCD) and an open-shell (RDCSD and UDCSD) implementation. ${ }^{35}$ The orbital-optimized and Brueckner versions can be amended by a subsequent perturbative F12 correction. ${ }^{77}$ Molecular properties and analytical nuclear gradients are implemented in Molpro for the closed-shell DCSD. Linear-scaling versions of DCSD are available in projected atomic orbital (PAO) and PNO implementations (cf. Secs. II D 2 and II D 3).

An extension of the distinguishable cluster approximation onto triple excitations (DC-CCSDT) ${ }^{84}$ is available through a GeCCo interface (vide infra) and as an ITF implementation. First benchmarks demonstrate superior accuracy of the method compared to the parent CCSDT method.

\section{EOM-CCSD and TD-CC2}

The equation-of-motion (EOM) CCSD excitation energies for the singlet excited states from the RHF reference can be calculated along with the excited-state density matrices and transition density matrices, according to the formulas described in Ref. 85 , from which first-order properties and oscillator strengths are obtained. The population analysis of the singles part of the excitation operator can be performed as well. By default, only excitation energies are calculated by solving the right-hand-side similaritytransformed Schrödinger equation. The iterative solution follows the recipe of the generalization of the Davidson procedure ${ }^{86}$ to nonsymmetric matrices. ${ }^{87}$ The EOM-CCSD code is generally blackbox, and it does not require any extra settings apart from the required number of states of a given symmetry. The calculation of properties requires approximately twice as much time due to the additional effort for solving the analogous left-hand-side equation, and therefore, it is performed on request only. The implementation of the EOM-CCSD method within the local approximation has been described in detail in Ref. 74. Ionization-potential (IP) and electron-attachment (EA) EOM-CCSD can be simulated by the EOM-CCSD code by introducing a diffuse orbital in the basis set (see, e.g., Refs. 88 and 89). The one-electron densities for excited states can be computed and stored for subsequent analysis. For EOM-CCSD transition densities, a Plasser-Lischka analysis in terms of charge-transfer numbers ${ }^{90}$ has been implemented in Molpro.

The canonical TD-CC2, as well as the ADC(2), with a "strict" treatment of transition moments [both independent from the TDLCC2 and LADC(2) codes of Kats et al., cf. Sec. II D 2] is also available within Molpro. ${ }^{91}$ Among application works utilizing the EOM-CCSD and ADC(2) codes, one can name the calculation of accurate UV-Vis spectra of thiophene ${ }^{92}$ and furan. ${ }^{93}$ The EOMCCSD method has been used for molecules as large as magnesium porphyrin.

\section{Molecular properties from CC wavefunctions}

Molecular properties in CC theory are usually derived as energy derivatives, which can conveniently be formulated through a Lagrangian formalism. ${ }^{95-97}$ First-order properties defined through this formalism are available in Molpro for CCSD,$^{98}$ QCISD, ${ }^{99}$ and $\operatorname{CCSD}(\mathrm{T})$ theories (along with corresponding gradients, see Sec. VI A). Since CC theory is nonvariational and, therefore, does not fulfill the Hellmann-Feynman theorem, the calculation of the expectation value of the corresponding perturbation operator gives a different alternative formalism for obtaining CC properties. ${ }^{100}$ The development of this formalism was hampered for some time by the appearance of the deexcitation $T^{\dagger}$ operators in the formula, and although some solutions to this problem were proposed, the most elegant solution is the expectation-value approach from Ref. 101, where an auxiliary excitation operator $S$, defined through the equation

$$
e^{S} \Phi_{0}=\frac{e^{T^{\dagger}} e^{T} \Phi_{0}}{\left\langle\Phi_{0} \mid e^{T^{\dagger}} e^{T} \Phi_{0}\right\rangle}
$$

is utilized to get rid of the infinite summation problem. The resulting formula for the first-order property $X$ is then given by the equation

$$
\langle X\rangle=\left\langle\Phi_{0} \mid e^{S^{\dagger}} e^{-T} \hat{X} e^{T} e^{-S^{\dagger}} \Phi_{0}\right\rangle .
$$

For Eq. (2), one can use the Baker-Campbell-Hausdorff (BCH) expansion and express it in terms of multiple commutators, whichgiven that both $T$ and $S$ operators are connected-shows that this formula is explicitly connected and additionally allows for a truncation of this formula preserving this condition. The formula has been implemented for the CCSD case with some approximations in the $S$ operators. ${ }^{102}$ Numerical tests show that most of these terms make negligible contributions to properties such as dipole moments and that for practical calculations, one can truncate at third order in $T$, which is the default option when requesting this property in Molpro.

An extension of this model to frequency-dependent secondorder properties has been proposed by Moszynski et al. ${ }^{103}$ for a general complex frequency $\omega$ and perturbation operators $X$ and $Y$ oscillating with $\omega$,

$$
\langle\langle X ; Y\rangle\rangle_{\omega}=\left\langle e^{-S} e^{T^{\dagger}} Y e^{-T^{\dagger}} e^{S} \Phi_{0} \mid \hat{\mathcal{P}}\left(e^{S^{\dagger}} T_{X}(\omega) e^{-S^{\dagger}}\right) \Phi_{0}\right\rangle+\text { g.c.c. },
$$

where $\hat{\mathcal{P}}$ is a superoperator which projects onto the 1-, 2-, etc., excitation space, $T_{X}(\omega)$ is the first-order response of the CC amplitudes to the perturbation $X$, and finally the abbreviation g.c.c. stands for the operation of replacing the frequency $\omega$ by $-\omega^{*}$ and taking the complex conjugate of the result. Based on this formula and employing the truncations up to $n$-tuple commutators, the polarization propagator at the coupled cluster level limited to single and double excitations has been derived and implemented in Ref. 104. Among several truncation levels proposed in this reference, the $\operatorname{CCSD(3)}$ one seems to be the most suitable one for routine applications. Its accuracy has been found to be at the same level as that of the CCSD Lagrangian formalism with unrelaxed orbitals, which can be easily deduced from the analysis of the leading order in terms of the fluctuation operator, which is the same in both cases. ${ }^{105}$

Since the polarization propagators can be calculated for any frequency, it is also possible to obtain intermolecular dispersion coefficients, such as $C_{6}$ and $C_{9}$, at the CCSD level by calculating several electric-dipole polarizability tensors at imaginary frequencies and by performing the Gauss-Legendre numerical quadrature. ${ }^{104}$ 


\section{B. Multireference methods}

\section{MCSCF and CASSCF}

The first second-order MCSCF methods in Molpro were developed by Werner and Meyer, ${ }^{106}$ who also contributed the first quadratically convergent state-averaged MCSCF method. ${ }^{107}$ A much improved implementation, which included an extension to CASSCF, was provided in 1985 by Werner and Knowles (WK). ${ }^{108,109}$ Due to the inclusion of higher-order terms in the orbital optimization, which account approximately for the periodicity of the energy in the orbital rotation parameters, and the inclusion of the orbitalCI coupling in the optimization, the Werner, Meyer, and Knowles (WMK) methods provide extremely fast and robust convergence. The CI optimization in the CASSCF methods is based on the efficient determinant-based full CI method of Knowles and Handy. ${ }^{11}$ Alternatively, spin-adapted configuration state functions (CSFs) can be used, in which case the coupling coefficients are computed on the fly by an efficient symmetric group technique. ${ }^{1}$ This also makes it possible to choose the CSF basis manually or to use restricted active space (RASSCF) wavefunctions. Recently, the convergence and reliability of the WMK method was further improved.

However, the computation time and memory requirements of second-order MCSCF methods grow strongly with the molecular size. This is due to the large number of two-electron integrals required for the orbital Hessian, namely, $(r s \mid k l)$ and $(r k \mid l s)$, where $k, l$ denote occupied orbitals and $r, s$ denote any orbitals. The number of these integrals scales as $O\left(N^{4}\right)$, and the time for their evaluation scales as $O\left(N^{5}\right)$, where $N$ represents the system size. To overcome this problem, a new MCSCF orbital optimization method has recently been proposed and implemented in Molpro, ${ }^{61}$ which combines a second-order optimization algorithm of the active orbitals with the first-order Super-CI (SCI) treatment of Roos et al. ${ }^{111,112}$ The active Hessian in this hybrid method (denoted as SO-SCI) can be built from the two-electron integrals $(r s \mid t u)$ and $(r t \mid u s)$ with two active orbitals $t, u$. Hence, the number of integrals only scales as $O\left(N^{2}\right)$ and the computation time scales as $O\left(N^{4}\right)$ (if the number of active orbitals is considered as independent of the molecular size). The orbitals and the CI coefficients are optimized alternately, yielding only first-order convergence. However, the convergence is substantially improved using the Limited Memory Broyden-Fletcher-Goldfarb-Shanno (L-BFGS) quasi-Newton method. ${ }^{113}$

In all test calculations so far, the SO-SCI method showed faster convergence than the SCI method, and this overcompensates the small additional cost introduced by the two-electron integral computation. The robust convergence of the SO-SCI method allows us to avoid a preceding Hartree-Fock calculation, if the character of the active orbitals is roughly known. Good starting guesses can be obtained by the "Automated Construction of Molecular Active Spaces from Atomic Valence Orbitals" (AVAS) ${ }^{114}$ with orbitals obtained from an atomic density guess. ${ }^{64}$

Table I shows examples for CASSCF calculations with the new first-order program for three large transition-metal complexes. ${ }^{67,115}$ Additionally, results with our local PNO-CASPT2 program ${ }^{62}$ (cf. Sec. II D 4) are presented. The structures of these complexes are depicted in Fig. 2. All CASSCF calculations were started with AVAS based on the Molpro atomic density guess orbitals. The largest CASSCF calculation included 5154 basis functions and took $12 \mathrm{~h}$ on a single computer node. More details about these computations can be found in Ref. 61.

The SO-SCI method can also be used for the optimization of single-determinant ROHF wavefunctions. Due to the secondorder optimization of the active orbitals, the SO-SCI method converges mostly faster and more robustly than the ROHF method, and we, therefore, recommend using the SO-SCI method, especially for large open-shell transition metal clusters where near degeneracies of several d-orbitals exist. Some examples for such calculations are presented in Table II.

The new CASSCF methods are well parallelized. An example for the speed-ups achieved with up to 20 cores for the CASSCF calculation for the cobalt-complex is shown in Fig. 1.

\section{Multireference configuration interaction}

All multireference methods in Molpro are fully or partially internally contracted (IC). This means that the configuration space is created by applying excitation operators to the complete fixed reference functions. ${ }^{116-118}$ The first fully contracted multireference configuration interaction (MRCI) program has been presented in 1981 by Werner and Reinsch. ${ }^{119,120}$ A much improved partially contracted MRCI method was implemented by Werner and Knowles (WK) in 1988. ${ }^{10,11}$ In this method, only the excitations with two electrons in the external (virtual) space are internally contracted. Nonvariational approximately size consistent approximations, such as the averaged coupled pair functional $^{121}(\mathrm{ACPF})$, variational perturbation theory (VPT) ${ }^{122}$ [equivalent to $\mathrm{CEPA}(0)]$, or quasi-degenerate variational perturbation theory (QD-VPT), ${ }^{123}$ are also available in Molpro's ICMRCI program. ${ }^{12}$ Somewhat later, the IC-MRCI method was extended to accurately treat electronically excited states. ${ }^{13}$ In order to avoid unphysical behavior near avoided crossings and

TABLE I. CASSCF and PNO-CASPT2 vertical excitation energies of the large transition metal complexes shown in Fig. 2. All results are calculated with the def2-tzvpp basis set. The timings of the ground state (GS) and the excited state (ES) CASSCF calculations [run on 15 (Fe, Ni) and 20 (Co) CPU cores] are shown as well. CASSCF results are from Ref. 61.

\begin{tabular}{lcccccccc}
\hline \hline Complex & Excitation & Active space & $N_{\text {atoms }}$ & Basis size & Time GS $(\mathrm{h})$ & Time ES (h) & $\Delta$ E CASSCF (eV) & $\Delta$ E PNO-CASPT2 (eV) \\
\hline $\mathrm{FeC}_{72} \mathrm{~N}_{2} \mathrm{H}_{100}$ & ${ }^{5} \mathrm{~A} \rightarrow{ }^{3} \mathrm{~A}$ & $\mathrm{CAS}(6,10)$ & 175 & 3785 & 5.6 & 3.4 & 1.972 & 1.897 \\
$\mathrm{Co}_{2} \mathrm{ON}_{4} \mathrm{C}_{70} \mathrm{H}_{106}$ & ${ }^{1} \mathrm{~A} \rightarrow{ }^{3} \mathrm{~A}$ & $\mathrm{CAS}(14,14)$ & 183 & 3937 & 8.2 & 8.3 & 0.009 & 0.333 \\
{$\left[\mathrm{NiC}_{90} \mathrm{~N}_{20} \mathrm{H}_{120}\right]^{2+}$} & ${ }^{3} \mathrm{~A} \rightarrow{ }^{1} \mathrm{~A}$ & $\mathrm{CAS}(8,10)$ & 231 & 5154 & 10.9 & 12.1 & 2.063 & 1.819 \\
\hline \hline
\end{tabular}




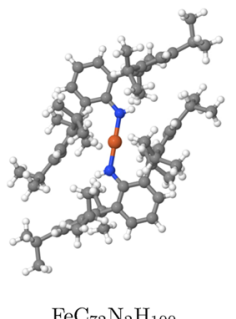

$\mathrm{FeC}_{72} \mathrm{~N}_{2} \mathrm{H}_{100}$

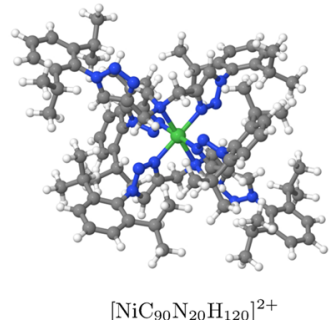

$\left[\mathrm{NiC}_{90} \mathrm{~N}_{20} \mathrm{H}_{120}\right]^{2+}$

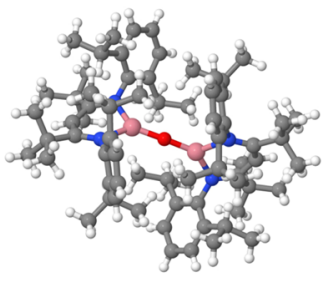

$\mathrm{Co}_{2} \mathrm{ON}_{4} \mathrm{C}_{70} \mathrm{H}_{106}$
FIG. 2. Transition metal complexes used in the calculations shown in Tables and II. conical intersections, the internally contracted configuration space has to be made invariant with respect to unitary transformations among the reference states. This can be achieved by using the union of the internally contracted configurations (ICCs) obtained from all (or some) reference states. With this ansatz, even narrow avoided crossings and conical intersections can be accurately described. However, the computational effort scales almost cubically with the number of states. This problem can be avoided by using state-specific ICCs. To avoid root-flipping problems, a projection technique can be used. ${ }^{13}$ At the end, a small CI in the space of the (non-orthogonal) $N_{\text {state }}$ wavefunctions $\left|\Psi_{n}\right\rangle$ is carried out, yielding finally orthogonal wavefunctions and variational energies for all included states. This works well unless the reference coefficients change strongly in the vicinity of narrow avoided crossings. In such cases, the contraction coefficients must be relaxed in order to obtain accurate potentials.

The WK ansatz described above avoids the computation and diagonalization of higher-order RDMs but has the disadvantage that the number of uncontracted CSFs increases strongly with the number of reference configurations. Furthermore, in cases with large active spaces, very many spin-couplings occur, many of which may have only a minor contribution since they do not belong to the first-order interacting space. In order to reduce these problems, Celani and Werner (CW) proposed to internally contract also parts of the internal and singly external configuration spaces. ${ }^{124}$ In the CW ansatz, all spaces that have at most two active orbital labels are contracted so that all required overlap matrices only depend on one- and two-electron RDMs. This greatly reduces the configuration space but has very little effect on the accuracy. In the original work of Celani and Werner, ${ }^{124}$ the CW ansatz was used for an efficient CASPT2 implementation denoted in Molpro RS2C.
Later on, a new IC-MRCI program (in Molpro called CIC) based on this ansatz has been developed by Shamasundar and Knizia. ${ }^{14}$ In both programs, all contributions of inactive (doubly occupied) orbitals are treated explicitly so that only the active-space RDMs are needed. This greatly improves the efficiency for larger molecules with many correlated inactive orbitals and eliminates a restriction to 32 correlated orbitals that is present in the original WK implementation. However, a huge number of terms in the amplitude equations occur. In the RS2C program, these were still implemented manually, but for MRCI (or even MRCC), this is not feasible. Therefore, a semi-automatic implementation based on the so-called Integrated Tensor Framework (ITF) developed by Knizia ${ }^{14,31}$ was used, which can efficiently execute long sequences of binary tensor contractions. The input is provided by a formula file, which can either be written manually or generated by a computer algebra program. The ITF program has been extended by Kats to support local domain and pair approximations ${ }^{62,125}$ (cf. Sec. II D 4). It has also been used to implement the QVCCD methods of Black et al. ${ }^{42,43}$ (cf. Sec. II A 3) as well as analytical MP2-F12 and CCSD(T)-F12 energy gradients, ${ }^{126,127}$ and it is currently used by Köhn and coworkers to implement a new efficient multireference coupled-cluster program.

Due to the high complexity, the CIC code currently only supports calculations with ICCs generated from a single-state reference wavefunction. However, we are actively working on multistate extensions and hope that these can be made available to the community in the near future.

\section{Multireference second-order perturbation theory}

Second-order multireference perturbation theory (MRPT2 or CASPT2) differs from non-variational variants of MRCI by

TABLE II. Open-shell single determinant calculations from Ref. 61 of the large transition metal complexes shown in Fig. 2. All calculations were done with the def2-tzvpp basis set and run on $15 \mathrm{CPU}$ cores. Density fitting was used in all cases for integral evaluation and transformation.

\begin{tabular}{|c|c|c|c|c|c|c|c|c|c|c|}
\hline \multirow[b]{2}{*}{ Complex } & \multirow[b]{2}{*}{ Basis size } & \multirow[b]{2}{*}{$N_{\text {occ }}$} & \multirow[b]{2}{*}{ State } & \multicolumn{2}{|c|}{ ROHF } & \multicolumn{2}{|c|}{ SCI } & \multicolumn{2}{|c|}{ SO-SCI } & \multirow[b]{2}{*}{ Energy (hartree } \\
\hline & & & & It. & Time (h) & It. & Time (h) & It. & Time (h) & \\
\hline $\mathrm{FeC}_{72} \mathrm{~N}_{2} \mathrm{H}_{100}$ & 3785 & 287 & ${ }^{5} \mathrm{~A}_{\mathrm{g}}$ & & $\mathrm{a}$ & 91 & 15.4 & 27 & 4.3 & -4156.211440 \\
\hline $\mathrm{Co}_{2} \mathrm{ON}_{4} \mathrm{C}_{70} \mathrm{H}_{106}$ & 3937 & 311 & ${ }^{7} \mathrm{~A}$ & 62 & 10.8 & 87 & 17.8 & 27 & 5.5 & -5768.892008 \\
\hline$\left[\mathrm{NiC}_{90} \mathrm{~N}_{20} \mathrm{H}_{120}\right]^{2+}$ & 5154 & 414 & ${ }^{3} \mathrm{~A}$ & 26 & 12.7 & 27 & 15.2 & 16 & 8.8 & -6074.841763 \\
\hline
\end{tabular}

${ }^{\mathrm{a}}$ No convergence after 100 iterations. 
replacing the full Hamiltonian in the Hylleraas functional by an effective one-electron Hamiltonian that only depends on a Fock matrix. Third-order multireference perturbation theory (MRPT3 and CASPT3) requires in addition effectively the computation of one MRCI residual. Thus, MRPT2 and MRPT3 programs can quite easily be obtained by modifying an MRCI program, skipping in the MRPT2 part all two-electron contributions and replacing the one-electron Hamiltonian by the effective Fock matrix. This has been done in the original RS2 and RS3 implementations in Molpro. ${ }^{128}$ For the reasons outlined in Sec. II B 2, these programs are less efficient than the more recent RS2C program of Celani, ${ }^{124}$ but they support multi-state (MS) CASPT2 calculations using the same wavefunction ansätze as described in Sec. II B 2 for the WK MRCI program. The proper treatment of conical intersections with MS-CASPT2 requires a configuration space that is invariant with respect to unitary transformations among the reference states. This has been realized in the extended multireference multi-state (MRXMS-CASPT2) method, ${ }^{129,130}$ which is based on the work of Granovsky. ${ }^{131}$ Recently, an approximate form of the XMS-CASPT2 has also been implemented, in which state-specific ICCs are used for each state (SR-XMS-CASPT2). Despite the fact that in this case the configuration space is not unitarily invariant with respect to rotation of the reference vectors, this approximation works quite well to describe avoided crossings where the coupling between the states is weak (e.g., the avoided crossing in LiF). However, larger discrepancies have been observed, for example, in the treatment of valenceRydberg mixings, and in this case, the MR-XMS-CASPT2 method is more reliable. A thorough investigation of these effects is currently being carried out and will be published elsewhere.

\section{Internally contracted multireference coupled-cluster theory}

Transferring the accuracy and size-consistency of a coupledcluster expansion of the wavefunction to the multireference domain has been one of the main challenges in electronic structure method development over the past decades; for reviews, see Refs. 132-134. A straightforward approach is to replace the reference determinant of conventional coupled-cluster theory by a multiconfigurational state,

$$
\left|\Psi_{n}\right\rangle=e^{\hat{T}} \sum_{R}\left|\Phi_{R}\right\rangle c_{R}^{n}
$$

where $\hat{T}$ is the cluster operator that, in addition to the usual occupied to virtual excitations, also involves excitations from the (doubly) occupied space into the active orbitals and from the active orbitals into the virtual space. As the ansatz generates internally contracted configurations, the notion internally contracted multireference coupled-cluster (icMRCC) theory has been established for this theory. Early work on this method was presented by Banerjee and Simons. ${ }^{135,136}$ The theory was recently revived ${ }^{137-140}$ and, in particular, extended by allowing for self-consistent relaxation of both cluster amplitudes and reference coefficients $c_{R}^{n}$. Relaxing the reference coefficients is in fact one of the main reasons for the particular accuracy of the resulting method, and it has been shown that it thus unifies state-specific and multistate coupled-cluster theories. ${ }^{141}$

The success of the method comes at a price of high complexity of the underlying equations, in particular, due to the noncommutativity of the cluster operator manifold. ${ }^{138}$ The current
Molpro implementation still relies on a symbolic code (called $\mathrm{GeCCo}$ ) that is generated by the automatized evaluation of the underlying second-quantized operator algebra. A more efficient implementation, based on the ITF and making use of Molpro features such as avoidance of the full integral transformation, is currently under development. At present, a convenient interface to Molpro is provided, which allows the transfer of all important run state variables (e.g., wavefunction symmetry and core orbitals) and to use procedures such as numerical geometry optimization. The methods icMRCCSD and icMRCCSD(T) can be selected directly from the Molpro input. A full integral transformation is performed, with the option to use density fitting, before the external program performing the icMRCC calculation is called.

Using this interface, a number of demonstrative applications have been carried out in the past, such as a highly accurate reactive potential energy surface for $[\mathrm{F}, \mathrm{H}, \mathrm{Cl}]^{142}$ or a set of benchmark computations for diatomic transition metal compounds. ${ }^{143}$ In order to address larger systems, an embedding scheme has been developed $^{144}$ that was inspired by the "region" approximation originally developed for $\operatorname{LCCSD}(\mathrm{T}) .{ }^{145}$ Here, a mean-field computation is carried out for the entire system, followed by localizing the orbitals using the intrinsic bond orbital (IBO) approach. ${ }^{146}$ From the full system, a local region is selected by both geometric and energy criteria, and the subsequent icMRCC computation is carried out for the subsystem only. Missing correlation contributions from the environment are added by a subtractive embedding approach, using the new PNO-CASPT2 program to treat the entire system. The approach is illustrated in Fig. 3.

Further work has been recently conducted in order to better understand the individual contributions to the icMRCCSD energy and to devise further approximations based on either perturbation theory ${ }^{147}$ or linearization of the equations. ${ }^{148}$ Excited states can also be targeted using icMRCC2 and linear response theory. ${ }^{149}$ All these efforts have the goal of providing a more efficient access to size-consistent multireference theories in future versions of Molpro.

\section{Uncontracted multireference and higher-order coupled cluster methods (MRCC)}

Molpro provides also an interface to the uncontracted MRCC program of Kállay et al. ${ }^{150}$ As the icMRCC program, this can be invoked using commands and options in the Molpro input. The program can also be used to carry out higher-order coupled-cluster calculations (including perturbative corrections and excitation energies).

\section{Explicitly correlated methods}

Explicitly correlated (F12) methods are available in Molpro for MP2, ${ }^{16,17}$ coupled cluster methods $[\operatorname{CCSD}(\mathrm{T})-\mathrm{F} 12 \mathrm{x}, \mathrm{x}=\{\mathrm{a}, \mathrm{b}, \mathrm{c}\}$ and DCSD-F12], ${ }^{18-20,35}$ as well as CASPT2 ${ }^{21}$ and MRCI. ${ }^{22-24}$ These methods greatly reduce the basis set incompleteness errors and are, therefore, strongly recommended for most calculations. When using F12, it is important to include diffuse functions in the basis set, and we recommend using the cc-pVXZ-F12 sets, ${ }^{151-154}$ which have been especially designed for F12 calculations. Such sets are also available for heavier elements with pseudo-potentials. ${ }^{15}$ 


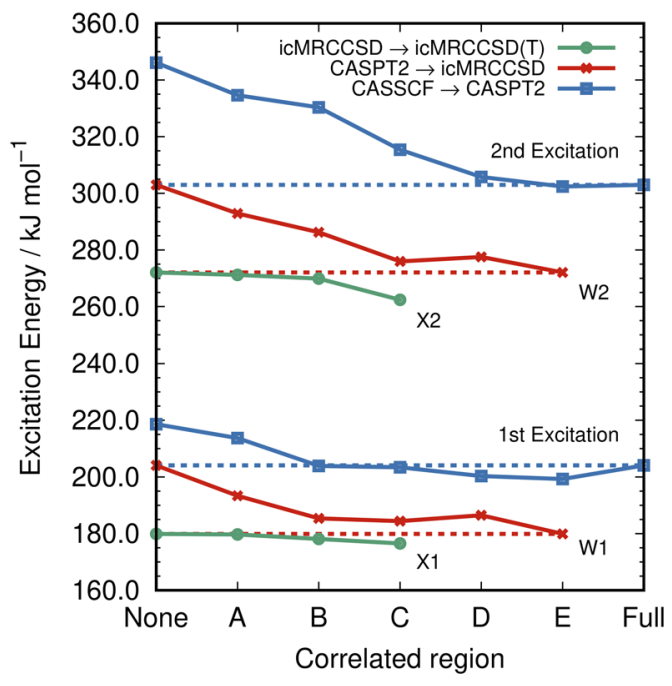

Region E

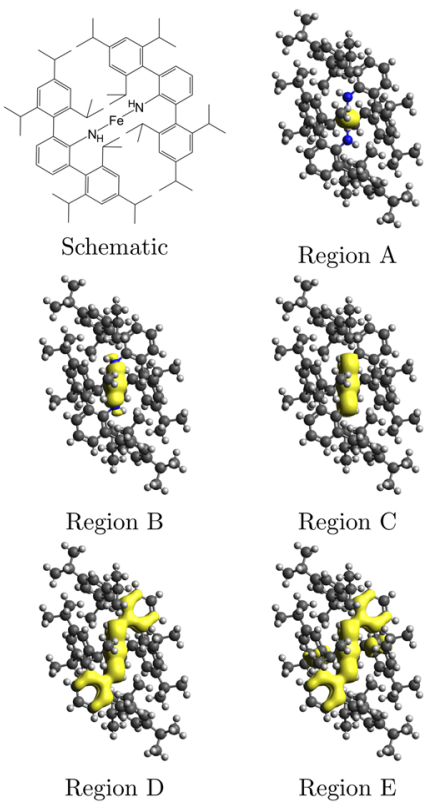

FIG. 3. Example of region-based embedding. Shown are the computed excitation energies (first excitation: $1{ }^{5} \mathrm{~A} \rightarrow 1{ }^{3} \mathrm{~A}$ and second excitation: $1{ }^{5} \mathrm{~A} \rightarrow 1^{1} \mathrm{~A}$ ) of an iron complex for different region sizes, as indicated in the right part of the figure. Reproduced with permission from Coughtrie et al., J. Chem. Theory Comput. 14, 693 (2018). Copyright 2018 American Chemical Society.
The application of F12 methods is very useful for highly accurate thermochemistry, ${ }^{19,35,155-157}$ including core-valence correlation effects, ${ }^{20,30,152}$ transition metal compounds, ${ }^{153,158}$ and intermolecular interactions. $^{26,27,159}$ In the latter case, it is recommended to use the recently added augmented aug-cc-pVXZ-F12 basis sets. ${ }^{160}$

In the F12 methods, all integrals that involve the F12 correlation factor are computed using density fitting approximations. Furthermore, the resolution of the identity (RI) approximation is necessary to approximate three-electron and four-electron integrals. Thus, apart from the orbital basis, also density fitting and RI auxiliary basis sets are necessary in F12 calculations. For most elements, these basis sets are chosen automatically. More details about these basis sets and the Molpro basis set library are given in Sec. VIII B.

In order to obtain highly accurate results with F12 methods, it is important to apply the complementary auxiliary basis set (CABS) singles correction ${ }^{18}$ in order to minimize errors of the Hartree-Fock energy contribution. This correction is applied automatically in all canonical F12 calculations. For explicitly correlated local correlation methods (cf. Sec. II D), the correction can either be computed separately or automatically. The default is to compute it separately since it does not include local approximations. Furthermore, local calculations are often repeated with different thresholds in order to study the convergence with respect to the local approximations, and since the CABS correction is additive, it is not necessary to recompute it each time.

\section{Local correlation methods}

\section{Orbital localization}

The occupied orbitals can be localized using the Foster-Boys, ${ }^{161}$ Pipek-Mezey, ${ }^{162}$ or IBO ${ }^{146,163}$ methods. The localization can be restricted to groups, as, for example, closed-shell and active orbitals.
The performance of local correlation methods as described below does not strongly depend on the choice of the localization procedure. A comparison can be found in Ref. 51 .

\section{PAO based CC methods}

In order to reduce the steep scaling of coupled cluster methods with system size, local correlation methods have been implemented in Molpro. The first generation of linear scaling methods in Molpro $^{164-180}$ used domains of projected atomic orbitals (PAOs) to span the local virtual orbital space for each electron pair. With these methods, energy calculations are possible up to the LCCSD(T)-F12 level, and analytical energy gradients are available for LMP2.

Excited state calculations for large systems, including the calculation of molecular properties and analytical nuclear gradients, can be done with local CC2 and ADC(2) methods. ${ }^{70-74,183,184}$ A problem of these approaches is that very large PAO domains are needed to approach the canonical limit closely, and it is difficult to obtain accurate and balanced results if the electronic structure of a system changes strongly during a reaction. This problem is much alleviated in the explicitly correlated variants since the explicitly correlated terms not only reduce basis set incompleteness errors but also strongly reduce the domain errors. ${ }^{44-47}$ Since 2012, a new generation of local coupled cluster methods has been developed, ${ }^{48-58}$ which are based on domains of pair natural orbitals (PNOs). These methods, which are much more accurate and efficient than PAO methods, are described in Sec. II D 3. At an intermediate stage, these methods may also use orbital specific virtual orbitals (OSVs). ${ }^{185-187}$

\section{Explicitly correlated local coupled-cluster methods using pair natural orbitals PNO-LCCSD(T)-F12}

Over the past few years, a new generation of local correlation methods using pair natural orbitals (PNOs) has been developed in Molpro, which brings roughly an order of magnitude reduction in local errors over the previous methods described in 
Sec. II D 2 at even lower costs. In Molpro2019, PNO-LMP2-F12, 49,50 and PNO-LCCSD(T)-F12 $2^{53-55}$ methods are available for closed-shell molecules. A review of these methods and extensive benchmark results can be found in Ref. 56. A new revision of the methods that supports both closed-shell and open-shell molecules, namely, PNORMP2-F12, ${ }^{57}$ PNO-RCCSD(T)-F12, and PNO-UCCSD(T)-F12, ${ }^{58}$ is featured in Molpro 2020 (the prefix "L" in the names of the methods is omitted for simplicity). Explicitly correlated distinguishable cluster methods (see Sec. II A 3) PNO-RDCSD-F12 and PNO-UDCSDF12 will also be available.

The local correlation methods are based on two distinct approximations: "domain approximations," which limit the correlation space for each electron pair to a domain of PAOs or PNOs, and "pair approximations." The latter simplify the amplitude equations for weak and distant pairs, which are very numerous, but each of them contributes only a very small amount to the total correlation energy. For example, distant pair energies can be approximated using multipole approximations, and the treatment of weak pairs can be simplified by linearizing the amplitude equations, neglecting small couplings between pairs, or neglecting terms in the amplitude equations that cancel at long range.

The major improvements over the PAO-based local methods include the following: (1) The use of PNOs on top of PAO domains leads to significantly more compact wavefunctions and, therefore, makes possible calculations on larger molecules with less aggressive local approximations. Using PNOs also allows the iterative solution of the local (T) equations at a reasonable cost. ${ }^{55}$ (2) Improved pair approximations in the LCCSD equations, ${ }^{53,188}$ together with more accurate multipole approximations for distant pair energies, ${ }^{52}$ are adopted. These techniques reduce the pair-approximation errors in relative energies to below $0.1 \mathrm{kcal} \mathrm{mol}^{-1}$ in nearly all systems we tested. (3) Near-linear scaling F12 treatment has been implemented. ${ }^{50,54}$ The F12 terms add only a small fraction to the computational cost yet significantly reduce the basis-set incompleteness and superposition errors, as well as local domain approximation errors. (4) Refined parameters for local approximations have been established based on systematic benchmark tests ${ }^{56}$ so that the methods can be used in a black-box manner. (5) Advanced parallelization techniques using both the Global Arrays toolkit ${ }^{189}$ and MPI (Message Passing Interface) point-to-point communication have been implemented to allow efficient cross-node parallelization on small computer clusters with fast network connection (e.g., InfiniBand) and hence a shorter time-to-solution.

As an illustration of the performance of the methods, we present some PNO-LCCSD(T)-F12 results in Tables III and IV. Calculations on systems involving open-shell molecules were computed with a development version of the PNO-RCCSD(T)-F12 program. Table III shows the errors of the local calculations against the canonical ones for several benchmark sets on small to medium sized molecules. The errors from the local approximations are only a small fraction of $1 \mathrm{kcal} \mathrm{mol}^{-1}$ for these systems using the default settings, and the errors can be further reduced by using large PAO and PNO domains. The pair approximations show little effect on the results. Table IV shows the results for some large and difficult test systems. It can be seen that the domain errors should be at the $1 \mathrm{kcal} \mathrm{mol}^{-1}$ scale, estimated from the difference between standard and large-domain calculations. All these large calculations can be done in hours of elapsed time on a small computer cluster. The largest PNO-LCCSD(T)-F12 calculation we have performed so far has more than 10000 basis functions, 300 atoms and 800 correlated electrons (using augmented triple- $\zeta$ basis sets).

Table $V$ presents some computed excitation energies for the Fe-complex in Fig. 2 using different methods and basis sets. For comparison, also the results from Table I are included. It is found that CASPT2, RMP2, and RCCSD yield very similar results, and also basis set effects are small. However, the inclusion of connected triple excitations lowers the excitation energies significantly.

\section{PNO-CASPT2}

The computational cost of the conventional CASPT2 method (cf. Sec. II B 3) scales as $\mathcal{O}\left(N^{5}\right)$ with the molecular size. However, the scaling can be reduced to linear by applying local correlation techniques, similar to the linear-scaling single-reference methods (cf. Secs. II D 2 and II D 3).

The PNO-CASPT2 method $^{62}$ is based on the internally contracted formalism, and therefore, the interacting configuration space can be divided into eight mutually orthogonal subspaces, coupled through generalized Fock matrices. These subspaces differ in the number of electrons in the closed-shell, active, and virtual orbital

TABLE III. Root-mean-square errors of PNO-LCCSD(T)-F12 relative energies against canonical CCSD(T)-F12 results in $\mathrm{kcal} \mathrm{mol}^{-1}$ using various program settings. Unless otherwise stated, the same F12 ansatz and basis sets were used in local and canonical calculations.

\begin{tabular}{lccc}
\hline \hline Benchmark set & Default & Large domains & Tight pair options \\
\hline Organic chemical reactions $^{\mathrm{a}}$ & 0.18 & 0.10 & 0.18 \\
Noncovalent interactions $^{\mathrm{b}}$ & 0.10 & 0.02 & 0.10 \\
Radical stability energies $^{\mathrm{c}}$ & 0.05 & 0.01 & 0.05 \\
Ionization potentials $^{\mathrm{d}}$ & 0.13 & 0.06 & 0.13 \\
\hline \hline
\end{tabular}

\footnotetext{
${ }^{a}$ A benchmark set of 51 reactions described in Ref. 190, cc-pVTZ-F12 basis.

${ }^{\mathrm{b}}$ S66 benchmark set, using the aug-cc-pVTZ-F12 basis and F12 scaled triples. Errors are against the "silver" CCSD(T)/CBS estimates in Ref. 191.

${ }^{\mathrm{c}}$ Radical stability energies of 30 radicals described in Ref. 192, aug-cc-pV(T+d)Z basis

${ }^{\mathrm{d}}$ Vertical ionization potentials of 30 ions described in Ref. 192, aug-cc-pVTZ basis.
} 
TABLE IV. PNO-LCCSD(T)-F12 reaction energies (in kcal mol ${ }^{-1}$ ) and computational cost of some reactions involving large molecules. The molecular size and computational time shown are for the most expensive single-point PNO-LCCSD(T)-F12 calculation in the reaction.

\begin{tabular}{|c|c|c|c|c|c|c|c|c|}
\hline \multirow[b]{2}{*}{ Reaction } & \multirow[b]{2}{*}{ Basis } & \multicolumn{3}{|c|}{ Largest calculation } & \multicolumn{2}{|c|}{ Reaction energy } & \multicolumn{2}{|c|}{ Cost (default settings) } \\
\hline & & Molecular formula & $N_{\mathrm{AO}}{ }^{\mathrm{a}}$ & $N_{\mathrm{el}}^{\mathrm{b}}$ & Default $^{\mathrm{c}}$ & Tight $^{\mathrm{d}}$ & No. of CPU cores & Elapsed time (h) \\
\hline ISOL $24-4^{e}$ & cc-pVTZ-F12 & $\mathrm{C}_{30} \mathrm{H}_{50} \mathrm{O}$ & 2543 & 176 & 67.5 & 68.0 & 80 & 3.6 \\
\hline AuAmin & cc-pVTZ-F12 & $\mathrm{AuN}_{4} \mathrm{PC}_{41} \mathrm{H}_{45}$ & 3345 & 244 & 47.3 & 47.7 & 120 & 6.4 \\
\hline WCCR10-4 & cc-pVTZ-F12 & $\mathrm{RuCl}_{2} \mathrm{P}_{3} \mathrm{C}_{62} \mathrm{H}_{106}$ & 5168 & 390 & 51.5 & 53.0 & 150 & 10.6 \\
\hline $\mathrm{Fe}(\operatorname{tacn})_{2}$ cplx. & aug-cc-pVTZ & $\mathrm{FeC}_{12} \mathrm{~N}_{6} \mathrm{H}_{30}$ & 1645 & 122 & 0.8 & 1.3 & 60 & 4.5 \\
\hline $\mathrm{FeC}_{72} \mathrm{~N}_{2} \mathrm{H}_{100}$ cplx. & aug-cc-pVDZ & $\mathrm{FeC}_{72} \mathrm{~N}_{2} \mathrm{H}_{100}$ & 2939 & 406 & 39.0 & 38.3 & 64 & 13.3 \\
\hline
\end{tabular}

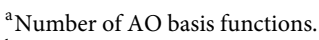

${ }^{\mathrm{b}}$ Number of correlated electrons.

${ }^{\mathrm{c}}$ Using default settings.

${ }^{\mathrm{d}}$ Using tight domain settings denoted as "domopt = tight" in Ref. 56. The local errors are almost exclusively from domain errors, and results using other settings are not shown here.

${ }^{\mathrm{e}}$ Reaction 4 from the ISOL24 set of isomerization reactions from Ref. 193.

${ }^{\mathrm{f}}$ Dissociation energy of a gold(I)-aminonitrene complex from Ref. 194, using re-optimized geometries from Ref. 49.

${ }^{\mathrm{g}}$ Dissociation energy of Complex 4 in the WCCR10 benchmark set from Ref. 195, using re-optimized geometries from Ref. 56.

${ }^{\mathrm{h} 1} A \rightarrow{ }^{5} \mathrm{~B}$ spin-state energy of $\left[\mathrm{Fe}(\operatorname{tacn})_{2}\right]^{2+}$, geometries from Ref. 196.

${ }^{\mathrm{i}}$ aug-cc-pwCVTZ basis for Fe. $3 s$ and $3 p$ orbitals of Fe are correlated.

${ }^{\mathrm{j} 5} \mathrm{~A} \rightarrow{ }^{3} \mathrm{~A}$ spin-state energy difference of an iron complex, geometries from Ref. 115.

subspaces. Some of them are expanded in PNO bases and others (involving single excitations) in the PAO basis. Due to the local restrictions, only the residual calculations for one of the eight subspaces scale linearly with the molecular size and all other residuals can be obtained in nearly constant time (cf. Fig. 4). It should be noted, however, that this applies to a linear chain system, for which the asymptotic region is quickly reached. For three-dimensional systems, the linear scaling region is much more difficult to reach. Nevertheless, very large systems can be treated in quite short times. The amplitude equations have been implemented using the local ITF framework, which can efficiently handle various local restrictions. ${ }^{62,125,197,198}$ A PNO-CASPT2 calculation of the iron-complex in Fig. 2 takes around 30 min using four compute cores.

Multiple states in the same space/spin symmetry can be obtained using state-averaged CASSCF, and the dynamical correlation can be added using the state-specific PNO-CASPT2 method. However, it is more efficient to use the multi-state PNO-CASPT2 method (PNO-MS-CASPT2), ${ }^{63}$ which additionally approximately

TABLE V. Energy differences (in $\mathrm{eV}$ ) between the triplet first excited state and the quintet ground state of the complex $\mathrm{FeC}_{72} \mathrm{~N}_{2} \mathrm{H}_{100}$. Basis aVTZ: aug-cc-pVTZ, $\mathrm{H}=\mathrm{cc}-\mathrm{pVTZ}$.

\begin{tabular}{lc}
\hline \hline Method & Energy difference \\
\hline HF/def2-TZVP & 2.496 \\
HF/aVTZ & 2.484 \\
CASSCF/def2-TZVPP & 1.972 \\
PNO-CASPT2/def2-TZVPP & 1.897 \\
PNO-RMP2/aVTZ & 1.888 \\
PNO-RMP2-F12/aVTZ & 1.778 \\
PNO-RCCSD-F12/aVTZ & 1.806 \\
PNO-RCCSD(T)-F12/aVTZ & 1.688 \\
\hline \hline
\end{tabular}

accounts for couplings of the states. A PNO-MS-CASPT2 calculation for 45 states for the same iron-complex takes about $12.5 \mathrm{~h}$ using four compute cores. Additional approximations are possible, which neglect couplings between some of the configuration subspaces. ${ }^{63}$ Then, the amplitudes which describe the correlation of the electrons in inactive orbitals (P2 space in Fig. 4) are identical for all states and need to be computed only once. Thus, the additional effort for computing the excited state energies is small and nearly independent of the molecular size. Using such approximations, the time for the abovementioned calculation with 45 states can be reduced to $7.2 \mathrm{~h}$, with a maximum additional error of $11 \mathrm{meV}$ in the excitation energies.

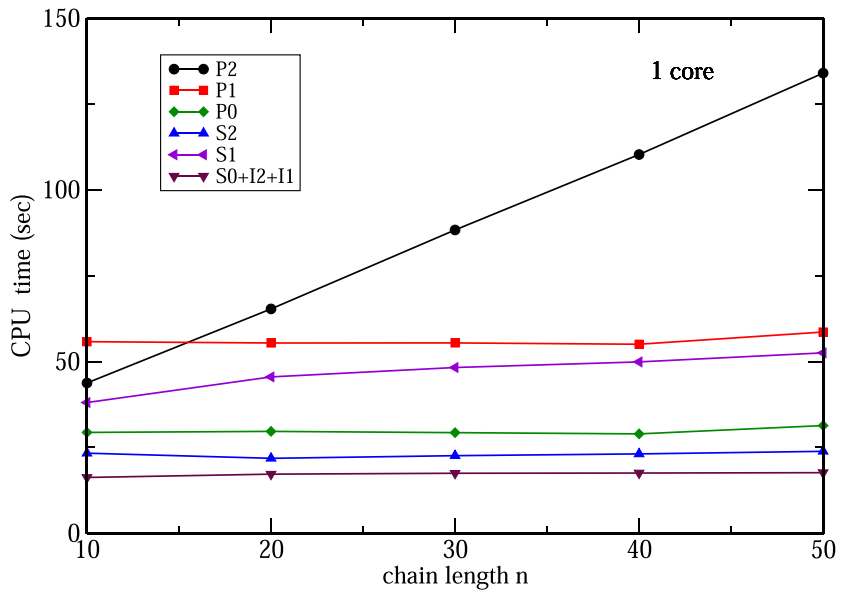

FIG. 4. Timings of individual contributions to the PNO-CASPT2 residuals for the ${ }^{1} A$ ground states of $\left[\left(\mathrm{C}_{4} \mathrm{SH}_{3}\right)-\left[\mathrm{CH}_{2}\right]_{n}-\left(\mathrm{C}_{4} \mathrm{SH}_{3}\right)\right]^{2+}$ using a $(10,10)$ active space. Reproduced with permission from Menezes et al., J. Chem. Phys. 145, 124115 (2016). Copyright 2016 AIP Publishing LLC. 


\section{DENSITY FUNCTIONAL METHODS}

Molpro implements Kohn-Sham theory in its most commonly used forms, including (a) pure density functionals that are integrals of general functions of the spin-density, its scalar gradient, and the kinetic energy density; (b) hybrid functionals that include also a fraction of the exact exchange energy; (c) additional empirical dispersion corrections including the $\mathrm{D} 3^{199}$ and $\mathrm{D} 4^{200}$ models and the nonlocal DFT (NLDFT) functional; ${ }^{201,202}$ (d) range-separated hybrids, with wavefunction methods for long-range correlation; (e) exact exchange (EXX) density functionals based on the local HartreeFock (LHF) method, ${ }^{203}$ and the optimized effective potential (OEP) method. ${ }^{20}$

Kohn-Sham theory in a finite basis requires evaluation of the exchange-correlation functional and its derivative with respect to elements of the one-particle density matrix. Numerical quadrature is implemented through a newly written library that generates grids for a pseudo-adaptive quadrature specified through a target accuracy and some other parameters. The usual fuzzy Voronoi partitioning $^{205,206}$ with a spherical-polar atomic coordinate system is used, and the library implements most of the available radial and angular quadrature schemes. A fixed-size radial quadrature is chosen, and then, at each radial grid point, experiments are carried out with different angular grids in order to achieve the target accuracy for a simple model energy density function with minimum effort. This scheme automatically ensures the avoidance of unnecessarily large grid densities close to nuclei (where a low-order spherical-harmonic expansion describes the density well) and distant (where electron density and/or Voronoi switch functions mean the integrand is small).

Density functionals are specified through a library in which the exchange-correlation energy density is defined using one or more Maple symbolic-algebra expressions. This library is then compiled into Fortran code using the dfauto ${ }^{207}$ utility, which also generates documentation.

\section{A. Dispersion corrections}

Common DFT methods need to be corrected by additional dispersion interaction energy contributions in order to describe noncovalent interactions between two separate molecules or to describe the intramolecular interactions of large molecular complexes. ${ }^{208}$ In addition to the standard D3 and D4 dispersion corrections by Grimme, ${ }^{199,200}$ Molpro contains an implementation of the NLDFT (nonlocal DFT) method that is based on a doubleHirshfeld partitioning of the correlation energy density. ${ }^{201,202}$ Due to this, the influence of the hybridization states of the interacting atoms on the long-range interaction is implicitly described, requiring no additional information of the atom bond partners like in the D3 model. ${ }^{199}$ Moreover, the NLDFT method is a consistent Kohn-Sham method because the long-range correction also describes a modified xc potential through the functional derivative of the NLDFT correlation functional. It was shown that the NLDFT method outperforms many dispersion corrected generalized gradient approximations (GGAs) and meta-GGA functionals for a large number of thermodynamic properties from the GMTKN30 database and it delivers mean absolute errors in the range of $0.2 \mathrm{kcal} / \mathrm{mol}$ only for the S22 and S66 benchmark databases. ${ }^{201,209,210}$ The accuracy of the method was also tested for describing the interaction energy of $\mathrm{H}_{2} \mathrm{O}$ with graphene, ${ }^{201}$ for the description of the interactions of large supramolecular complexes ${ }^{211}$ and for the description of conformer geometries of a range of peptide molecules. ${ }^{202}$

\section{B. Exact exchange Kohn-Sham methods}

Unlike in common hybrid Kohn-Sham (KS) DFT methods, in exact exchange (EXX) KS methods, the functional derivative with respect to the exact exchange energy is taken with respect to the electron density, ${ }^{212,213}$

$$
v_{\mathrm{x}}(\mathbf{r})=\frac{\delta E_{\mathrm{x}}[\rho]}{\delta \rho(\mathbf{r})},
$$

yielding a local exchange potential. Due to this, EXX methods have a number of advantages as compared to the Hartree-Fock method, e.g., orbital energy differences computed from EXX orbital energies are suitable first approximations to valence excitation energies (see, e.g., Ref. 214). Molpro contains various EXX implementations based on the optimized effective potential (OEP) method ${ }^{204,215-218}$ and the common energy denominator approximation (LHF and CEDA methods). ${ }^{203,219,220}$ Orbitals from EXX or hybrid-EXX (mixing the EXX functional with standard GGA exchange and correlation functionals) calculations are also suitable for use in DFT-SAPT calculations (see Sec. VII B 1) because the local exact exchange potential $v_{x}(\mathbf{r})$ possesses the correct asymptotic behavior and thus yields an improved description of the electronic density in the asymptotic range. $^{221}$

\section{Time-dependent density functional theory}

A basic implementation of time-dependent density functional theory (TDDFT) ${ }^{222}$ is available in Molpro that can be used to calculate excitation energies and linear response properties within the standard GGA. While having a number of limitations regarding choices for the exchange-correlation functional and kernel, the program is capable to treat large systems through the employment of density-fitting techniques for computing the Hessian-times-vector products.

Exact-exchange TDDFT (TDEXX) calculations ${ }^{214,223-225}$ can currently be done within the adiabatic approximation to the TDEXX kernel. This feature may be used in conjunction with EXX groundstate calculations (see Sec. III B).

The response module of the TDDFT program can be used both for computing static and frequency-dependent linear response properties for arbitrary one-electron operators available in Molpro and for computing the coupled Kohn-Sham response matrix of the molecule at a given (imaginary) frequency. These quantities can be used to calculate two-body and three-body dispersion interaction energies between two/three noncovalently bonded subsystems. The former is required, e.g., to calculate the coupled and uncoupled dispersion energies in MP2C calculations (see Sec. VII A 2).

\section{MULTISCALE MODELLING}

\section{A. Quantum mechanics/molecular mechanics}

Combined quantum mechanics/molecular mechanics (QM/ $\mathrm{MM}$ ) calculations are possible using practically any electronic 
structure method in Molpro for the QM region. The key elements of the interface are the lattice and force commands, respectively, allowing an array of point charges to be specified and forces on the QM atoms to be computed. The charges are specified in a simple file format in which each row specifies the Cartesian coordinates of the charge (in $\AA$ ), the charge itself, and a flag to indicate whether the gradient with respect to the coordinates of this charge should be computed. Interfacing to MM molecular dynamics packages can be hand-rolled using these tools, or there is also an existing Molpro interface in the Tcl-ChemShell package. ${ }^{226}$

For QM/MM calculations with large MM regions, the interaction contribution can become a bottleneck. In Molpro, extremely rapid evaluation of these contributions is achieved by unpublished early-summation techniques (inspired by analogous approaches for the Coulomb operator ${ }^{227,228}$ ). For the energy, the Boys function can be contracted immediately with the fixed point charges so that the recurrence relations that build and transfer angular momentum, and the contraction steps, are performed only once, not once per point charge. Similarly, in the gradient, early contraction with the density (as is commonly done) prevents the need to compute individual gradient integrals.

The density-fitted local correlation methods and QM/MM functionality in Molpro have been combined in numerous studies in enzymology. ${ }^{229-232}$ More recently, and as described in Sec. IV B, three-layer combinations of wavefunction-based methods, density functional theory, and MM have proved a powerful addition to the suite of tools available in Molpro for the treatment of complex, multiscale chemical problems.

\section{B. Projection-based wavefunction-in-DFT embedding}

Despite dramatic improvements in algorithms and efficiency, it is often the case that the sheer complexity of the chemical problem precludes direct application of coupled-cluster or multireference methods. For such problems, DFT plays a key role, but selection of the exchange-correlation functional can be difficult, and systematic deficiencies across large classes of functionals can lead to errors that are hard to eliminate.

Molpro provides a suite of tools that allow for multiscale combinations of highly accurate wavefunction-based methods with DFT. This allows high-accuracy treatment of a small, chemically relevant part, with a more approximate description of a complex chemical environment. Wavefunction-in-DFT (WF-in-DFT) embedding in Molpro is based on the use of projection operators to maintain orthogonal subsets of orbitals describing the two subsystems. ${ }^{59}$ This simple approach is robust and efficient, leading to a total computational cost equivalent to DFT on the whole system, plus the high-level calculation on the small, active subsystem. The coupling between subsystems is entirely captured through a modified coreHamiltonian in the high-level subsystem so that almost any electronic structure method in Molpro can be used. The implementation in Molpro has been tested and applied in a range of application areas, ${ }^{233-240}$ demonstrating the robustness and utility of the approach.

The method was used in combination with experiment in a detailed study of a new class of cobalt-based hydrogen-evolution catalysts. ${ }^{235}$ A small subsystem, containing the transition metal and its first coordination sphere, was treated using OSV-LCCSD(T), ${ }^{186,187}$ with the surrounding environment described using DFT with the BP86 functional. This combination provided close agreement with $\operatorname{LCCSD}(\mathrm{T})$ on the whole complex, while the restriction of the coupled-cluster treatment to a small, electronically complex region reduced the computational cost of each energy evaluation by a factor of around 20.

DFT-often in combination with QM/MM-is a standard workhorse for computational enzymology. Elucidation of reaction mechanisms requires reliable prediction of barrier heights across competing chemical processes, and conclusions from DFT are often sensitive on the choice of the exchange-correlation functional. Projection-based embedding combining CCSD(T) on a small, active subsystem with DFT on the surrounding chemical environment all but eliminates dependence on the choice of functional. ${ }^{236,239}$

New features that greatly improve the range of applicability of projection-based embedding include efficient truncation of the atomic-orbital basis set for the high-level calculation ${ }^{241}$ and development of analytic gradients for CCSD(T)-in-DFT. ${ }^{242}$ Robustness of calculations can further be improved using the even-handed approach for partitioning the system into high- and low-level subsystems. $^{238}$ Together, these provide a powerful tool for studying reactivity in complex chemical environments.

\section{RELATIVISTIC EFFECTS}

\section{A. All-electron effective Hamiltonians}

For all-electron calculations, Molpro allows the use of the Douglas-Kroll-Hess (DKH) ${ }^{243-245}$ and eXact-2-Component (X2C) scalar relativistic Hamiltonians. ${ }^{246,247}$ In both cases, only the oneelectron integrals are modified. In the case of the DKH Hamiltonian, the default order of the associated unitary transformation is 2, but arbitrarily higher orders can be selected. ${ }^{248}$ Because scalar relativistic effects change, in particular, the radial extent of inner-core orbitals, it is important to use orbital basis sets that are optimized using the relativistic Hamiltonian; as described in Sec. VIII B, these are available for most of the periodic table. Related to the use of these all-electron relativistic Hamiltonians, Molpro now also allows the use of a Gaussian finite nuclear model ${ }^{249}$ instead of the default point-charge nucleus.

\section{B. Pseudopotentials}

Effective core potentials (ECPs) or pseudopotentials (PPs) can be an effective and convenient way to include relativistic effects, both scalar and spin-orbit. Of course, in many cases, the use of PPs can also lead to significant computational cost savings due to the reduced number of electrons and basis functions included in the calculation. This becomes less of a factor, however, when highly correlated methods such as coupled cluster or MRCI are used. The implementation of a given pseudopotential $V_{p p}$ in Molpro is based on the following general form involving a maximum of four terms: $:^{250}$

$$
\begin{aligned}
V_{P P}= & -\frac{Z-n_{\text {core }}}{r}+V_{\ell_{\max }}+\sum_{\ell=0}^{\ell_{\max }-1}\left(V_{\ell}-V_{\ell_{\max }}\right) \mathcal{P}_{\ell} \\
& +\sum_{\ell=1}^{\ell_{\max }^{\prime}} \Delta V_{\ell} \mathcal{P}_{\ell} \boldsymbol{\ell} \cdot \mathbf{s} \mathcal{P}_{\ell},
\end{aligned}
$$


corresponding to (i) the monopole potential with core charge $Q=Z-n_{\text {core }}$, where $n_{\text {core }}$ is the number of core electrons replaced by the PP, (ii) a local term, (iii) a semi-local term for scalar relativity, and (iv) a semi-local term for the radial potentials of the spin-orbit (SO) part. The semi-local terms involve angular momentum projectors $\mathcal{P}_{\ell}$, and the terms involving $V_{\ell_{\max }}, V_{\ell}-V_{\ell_{\max }}$, and $\Delta V_{\ell}$ are defined by expansions over terms $c_{j} r^{m_{j}-2} e^{-\gamma_{j} r^{2}}$ with tabulated parameters $c_{j}, m_{j}$, and $\gamma_{j}$. The one-electron SO term, if defined, can be used in the state-interacting approach as described above.

A large number of PP families are available from the Molpro basis set library. These include the LANL ECPs, ${ }^{251,252}$ the SBKJC series, ${ }^{253,254}$ the Casino ECPs, ${ }^{255,256}$ and the energy-consistent PPs from the Stuttgart/Köln groups. ${ }^{257,258}$ Other PPs can easily be entered directly into the input as long as they adhere to the general form above. An ECP for a given element generally has one or more Gaussian basis sets associated with it-often just of doublezeta quality - but in the cases of many of the Stuttgart/Köln PPs, the latter are accompanied by full sequences of correlation consistent basis sets, e.g., cc-pVnZ-PP ( $n=D, T, Q, 5)$. See, for instance, Ref. 259. It is important to note that the resulting accuracy of a particular choice of ECP, as compared to an analogous scalar relativistic all-electron calculation, for example, is not only dependent on the underlying adjustment method used to define the ECP and the number of core electrons it replaces but also on the basis set used to represent the remaining valence electrons. In general, a given basis set is also not transferable between different ECPs.

\section{Spin-orbit coupling}

The calculation of spin-orbit coupling is implemented for MRCI wavefunctions using the Breit-Pauli operator. For any atoms described with a PP, the one-electron spin-orbit part of PP replaces the bare-nucleus operator. Matrix elements of the operator between MRCI wavefunctions are evaluated for a single choice of $\hat{S}_{z}$ eigenvalue, and then, the complete effective Hamiltonian matrix is constructed using the Wigner-Eckart theorem. Diagonalization of this Hamiltonian yields new states, for which properties such as dipole matrix elements are transformed. To make calculations more efficient without significant sacrifice of accuracy, an effective Fock matrix technique is used for the external parts of the MRCI wavefunction but retaining the full two-electron operator for the dominant part of the wavefunction with configurations that occupy only internal orbitals. ${ }^{260,261}$

For larger systems, different approximate schemes are implemented. These include the use of effective Fock matrix for internal parts as well (then spin-orbit becomes one-electron effective operator) and the use of one-center approximation. In these cases, the integral-direct calculations can strongly speed up the computation.

\section{MOLECULAR PROPERTIES AND WAVEFUNCTION ANALYSIS}

\section{A. Energy gradients, geometry optimization, and harmonic vibrational frequencies}

Analytical gradients are implemented for Hartree-Fock (spin restricted and unrestricted), DFT, and most single reference methods. This includes closed-shell MP2, ${ }^{175,181,182}$ CCSD, DCSD,
QCISD(T), ${ }^{99}$ and $\operatorname{CCSD}(\mathrm{T})$ as well as for the corresponding F12 methods. ${ }^{126,127}$ Particularly accurate geometries are obtained with DCSD-F12 gradients, ${ }^{35}$ which avoids the expensive inclusion of triple excitations.

Analytical MCSCF and CASPT2 gradients ${ }^{129,262,263}$ are available for the methods based on the WK contraction (cf. Secs. II B 2 and II B 3), including MS-CASPT2 and XMS-CASPT2. ${ }^{129,263}$ For most methods, the gradients are implemented with and without density fitting approximations. The latter are highly efficient and preferable for most applications. Gradients are also available for local MP2, ${ }^{175,181,182}$ using either Pipek-Mezey localization ${ }^{162}$ or intrinsic bond orbitals (IBOs). ${ }^{146}$

Geometry optimizations ${ }^{264,265}$ and harmonic frequency calculations use the analytical gradients whenever available. Otherwise, the gradients or Hessians are computed automatically using finite difference methods. In such numerical derivative calculations, the energies or gradients for different displacements can be computed in parallel. The program automatically switches from its normal mode (parallel execution of a single workflow) to devolve the displacedgeometry energies to each MPI process as a serial task; this embarrassingly parallel strategy leads to very good parallel scaling of the overall derivative computation.

\section{B. Anharmonic vibrational spectra}

Besides the multitude of electronic structure methods, Molpro offers a variety of programs for solving the nuclear Schrödinger equation, all of them being based on the Watson Hamiltonian. ${ }^{266}$ This allows for the very accurate simulation of vibrational and vibronic spectra as well as many vibrationally averaged properties.

The first step within these calculations is the generation of a multidimensional potential energy surface (PES), which can be obtained in a fully automated manner using any of the implemented electronic structure methods. ${ }^{267,268}$ Likewise, dipole moment and polarizability tensor surfaces as needed for infrared and Raman intensities can be generated for those methods, for which analytical gradients are available. ${ }^{269}$ Usually, these surfaces are represented in an $n$-mode expansion of a user-defined order. ${ }^{270}$ Alternatively, one may use quartic or sextic force fields. The PES generator can handle any molecular point group and can exploit molecular point group symmetry and permutational symmetry in dependence on the chosen coordinate system, which is currently restricted to canonical or localized normal coordinates. ${ }^{268,271}$ Multi-level schemes ${ }^{272,273}$ and interpolation and prescreening techniques ${ }^{271}$ in combination with an embarrassingly parallel implementation allow for the very efficient calculation of PESs of local minima or degenerate doublewell potentials. ${ }^{274}$ In particular, the modeling of high-order terms of the PES expansion leads to substantial accelerations. ${ }^{275,276}$ These potentials can be dumped as ASCII files to be used in any other program. In a subsequent transformation program, the grid representation of the PES can be transformed to an analytical sumof-products representation of multivariate polynomials, B-splines, or distributed Gaussians. ${ }^{277}$ This transformation is based on highly efficient Kronecker product fitting.

Once the PES has been generated, it can be used for determining wavefunctions from conventional vibrational selfconsistent field theory (VSCF), configuration-averaged VSCF theory 
(CA-VSCF) ${ }^{278}$ or multiconfigurational self-consistent field theory (VMCSCF). ${ }^{279-281}$ Grid-based and finite basis variants of these programs are available. One-mode wavefunctions (modals) are expanded in terms of harmonic oscillator functions or modespecific, local distributed Gaussians. Vibrational angular momentum terms are added $a$ posteriori to the state energies. ${ }^{282}$ In order to account for vibration correlation effects, programs for 2nd order vibrational Møller-Plesset perturbation theory (VMP2), vibrational configuration interaction (VCI) calculations, or vibrational multireference configuration interaction theory (VMRCI) have been implemented. ${ }^{283-285}$ These programs can handle up to 9-tuple excitations and provide a variety of options to tailor the configuration space. Vibrational ground-state and state-specific calculations are offered, which fully exploit molecular symmetry, even for non-Abelian point groups. By default, the VCI program uses a highly efficient configuration selection scheme, which allows for calculations of up to $10^{10}$ initial configurations. ${ }^{283,286}$ Sparse matrix techniques and a newly developed eigenvalue solver are used to limit memory demands and to speed up the calculations. ${ }^{287}$ Once the PES has been determined from explicitly correlated coupled-cluster calculations and the $n$-mode expansion of the PES has been converged, typical mean absolute deviations are in the range of $1-5 \mathrm{~cm}^{-1}$ for fundamental transitions and slightly larger values for overtones and combination bands. ${ }^{288}$ For example, Table VI shows the mean absolute and maximum deviations of computed VCI fundamentals from experimental gas phase or matrix isolation data for a small set of molecules. Molecular properties, such as intensities, vibrationally averaged rotational constants, molecular geometries, and dipole moments, can be obtained from these VCI calculations. ${ }^{289}$ These programs have been applied to a multitude of molecules and molecular clusters of up to 20 atoms. ${ }^{286}$ Besides these variational methods, a program based on 2nd order vibrational perturbation theory (VPT2) is available, which relies on the polynomial coefficients determined in the PES transformation program. ${ }^{277,290}$

Photoelectron or photoionization spectra can be computed in two different ways. This first one being offered by Molpro relies on a selection of Franck-Condon factors at the VSCF level within a sumover-states approach. ${ }^{291}$ In order to match the normal coordinates of the two potential energy surfaces, a Duschinsky transformation is provided, which can also be used to alter the normal coordinates of a PES in order to compute vibrational spectra for any isotopologues without recomputing the PES. ${ }^{292}$ Once the list of significant FranckCondon factors has been generated, the corresponding vibrational states can be computed at the VCI level, which will be used for

TABLE VI. Mean absolute (MAD) and maximum deviations (MAX) of VCl calculations based on explicitly correlated coupled-cluster calculations from experimental data for the fundamental modes of a small set of molecules (in $\mathrm{cm}^{-1}$ ).

\begin{tabular}{lcc}
\hline \hline Molecule & MAD & MAX \\
\hline $\mathrm{CH}_{2} \mathrm{~F}_{2}$ & 1.6 & 4.3 \\
$\mathrm{C}_{2} \mathrm{H}_{4}$ & 2.7 & 5.9 \\
$\mathrm{CH}_{3} \mathrm{OPO}$ & 4.4 & 8.2 \\
$\mathrm{C}_{2} \mathrm{H}_{6}$ & 1.5 & 3.6 \\
$\mathrm{CH}_{3} \mathrm{CH}_{2} \mathrm{~F}$ & 2.1 & 7.4 \\
\hline \hline
\end{tabular}

the calculation of Franck-Condon factors at the correlated level. Note that this approach is only meaningful once the vibrational states retain their state identity within the VCI calculations; otherwise, the sum of the Franck-Condon factors will not add up to 1.0. This approach has been applied to a variety of small molecules, e.g., ketene. ${ }^{293}$ In cases of high density of states or the loss of the state identity in general, the time-independent Raman wavefunction approach (RWF) ${ }^{294}$ can be used instead, which avoids the explicit calculation of vibrational eigenstates altogether but determines the orientationally averaged absorption cross section at relevant spectral points. This leads to an iterative solution of the inhomogeneous Schrödinger equation, for which several techniques have been developed. This method was found to be very robust, even for very high state densities; see, for example, the spectrum of $\mathrm{CH}_{2} \mathrm{~F}_{2} .{ }^{295}$ Quite recently, this approach has been extended for the calculation of Herzberg-Teller terms.

\section{One-electron properties and transition moments}

Normally, the expectation values and transition moments of standard one-electron properties are computed automatically where possible. By default, only dipole moments are evaluated, but other properties can be requested, including multipole moments, electric field and its gradient, angular momentum, diamagnetic shielding tensor, and velocity. Transition properties can be calculated for state-averaged CASSCF calculations or for MRCI wavefunctions. For MRCI wavefunctions, it is also possible to evaluate properties in the case when bra- and ket-molecular orbitals are different, via a transformation to bi-orthogonal orbitals. ${ }^{296}$ Calculation of overlaps, one-electron transition properties, and Hamiltonian operators is possible, including the overlap between MRCI wavefunctions obtained at different molecular geometries. These can be used, by applying numerical differentiation, to obtain non-adiabatic firstand second-order corrections.

\section{Population analysis, IBO, and NBO}

Mulliken population analysis and distributed multipole analysis $^{297}$ as well as intrinsic bond orbital (IBO) analysis ${ }^{146}$ are available in Molpro. The latter is based on Pipek-Mezey localized orbitals in a polarized minimal basis of atomic orbitals that spans the HartreeFock wavefunction exactly. The results of this method are particularly independent of the basis set and give a picture that corresponds closely to chemical intuition. Some applications can be found in Refs. 298-302. Molpro also provides an interface to the NBO6 program.

\section{INTERMOLECULAR INTERACTIONS}

\section{A. Supermolecular calculations}

Intermolecular interaction energies can be computed either using supermolecular calculations or by symmetry adapted perturbation theory (SAPT). The former has the advantage that any electronic structure method can be used, and the current gold standard for such calculations is $\operatorname{CCSD}(\mathrm{T})$ or, even better, $\operatorname{CCSD}(\mathrm{T})$ F12. ${ }^{191}$ On the other hand, SAPT calculations make it possible to 
analyze the interaction energies and to determine various physical meaningful contributions. Larger systems, for which canonical $\operatorname{CCSD}(\mathrm{T})$ calculations are too expensive, can be treated very accurately using the new PNO-CCSD(T)-F12 program in Molpro. For recent benchmarks and references to previous work, see Ref. 159.

\section{Counterpoise corrections}

Supermolecular calculations of interaction energies require counter-poise (CP) corrections ${ }^{304}$ to compensate the basis set superposition errors (BSSEs). This is even recommended with explicitly correlated methods which yields results close to the complete basis set (CBS) limit already with triple- $\zeta$ basis sets. Counterpoise calculations can be straightforwardly done by specifying "dummy" atoms, which carry a basis set but no nuclear charge and no electrons. It is also possible to remove the basis set from the dummy atoms for convenient computation of the monomer energies at formally infinite separation.

\section{Dispersion corrections to MP2}

The standard second-order Møller-Plesset perturbation theory (MP2) method fails to describe long-range correlation energies accurately. The reason for this stems from the fact that the MP2 method does not take into account intramolecular correlation effects of two interacting monomers, i.e., describes dispersion interactions on an uncoupled Hartree-Fock (UCHF) level. ${ }^{305,306}$ To remedy this, the supermolecular MP2 interaction energy can be corrected in a hybrid supermolecular-perturbation theory approach by subtracting the uncoupled HF (UCHF) dispersion energy and adding the dispersion energy from a more accurate response theory method instead. ${ }^{306,307}$ In the MP2C (MP2 coupled) method, the latter is computed using the time-dependent density-functional theory (TDDFT) approach, employing static response functions from the EXX method (see Sec. III B) and coupled response functions computed by using the adiabatic local density approximation (ALDA) exchange kernel,

$$
\Delta E_{\mathrm{int}}^{\mathrm{MP} 2 \mathrm{C}}=\Delta E_{\mathrm{int}}^{\mathrm{MP} 2}-E_{\text {disp }}^{(20)}[\mathrm{UCHF}]+E_{\text {disp }}^{(2)}[\mathrm{TDDFT}] .
$$

In Molpro, MP2C calculations can be performed with the aid of the TDDFT module that computes frequency-dependent coupled or uncoupled response functions. Exact-exchange KS calculations required to compute $E_{\text {disp }}^{(2)}$ [TDDFT] can be performed using the EXX methods described in Sec. III B. A remarkable feature of the MP2C approach of Eq. (7) is that it delivers far more accurate interaction energies than the MP2 method for dispersion energy dominated systems, while in the case of hydrogen-bonded systems, the overall well performance of MP2 is conserved. ${ }^{307,308}$ In a thorough study of Burns et al. of a large number of different spin-componentscaled (SCS), explicitly correlated (F12), and specialized wavefunction approaches using four different benchmark data bases with 94 dimers and 345 geometry configurations in total, the MP2C method was appointed to bronze standard for describing noncovalent interactions, as it yields very accurate energies at a moderate computational cost as compared to the other methods studied. ${ }^{309}$ Binding energies of large supramolecular complexes were found to be very similar to results from DFT-SAPT (Sec. VII B 1) or the NLDFT method (Sec. III A). ${ }^{211}$

\section{B. Symmetry adapted perturbation theory (SAPT)}

\section{Symmetry-adapted perturbation theory based on the Kohn-Sham description of monomers}

Intermolecular interaction energies between two noncovalently bonded molecules (denoted as monomers) can be calculated by perturbation theory approaches using the product of the monomer wavefunctions as the zeroth order approximation to the dimer wavefunction. In order to enforce the proper antisymmetry of the dimer wavefunction, various weak to strong symmetry forcing procedures can be employed, ${ }^{310}$ which are generally known under the name symmetry-adapted perturbation theory (SAPT). ${ }^{310-312}$ Among these methods, the symmetrized Rayleigh-Schrödinger theory (SRS), which belongs to the weak symmetry-forcing group, turned out to be the best practical choice as it yields very accurate intermolecular interaction energies at equilibrium and even in the short distance range. ${ }^{310,313}$ The SAPT interaction energy is typically expanded up to second order in terms of the intermolecular interaction operator (defined as the difference between the total electronic Hamiltonian of the dimer and the sum of monomer Hamiltonians), leading to the terms

$$
\Delta E_{\text {int }}^{\mathrm{SAPT}}=E_{\text {pol }}^{(1)}+E_{\text {exch }}^{(1)}+E_{\text {ind }}^{(2)}+E_{\text {exch-ind }}^{(2)}+E_{\text {disp }}^{(2)}+E_{\text {exch-disp }}^{(2)}+\Delta^{3-\infty}
$$

with $E_{p o l}^{(1)}$ as the electrostatic energy, $E_{\text {ind }}^{(2)}$ as the induction energy, $E_{\text {disp }}^{(2)}$ as the dispersion energy, and $E_{\text {exch }}^{(1)}, E_{\text {exch-ind }}^{(2)}, E_{\text {exch-disp }}^{(2)}$ are the corresponding exchange interaction counterparts that stem from a tunneling of the electrons among the monomers and which vanish at large distances between monomers. The term $\Delta^{3-\infty}$ defines an approximation to the higher order interaction energy terms and can be estimated using the supermolecular Hartree-Fock method. ${ }^{314}$ As compared to the supermolecular method, the SAPT method has a number of advantageous features. Namely, it is free from any basis set superposition error (BSSE) and it yields a deeper physical insight into the nature of the interaction through the energy decomposition of Eq. (8)

In order to obtain accurate interaction energies from Eq. (8), intramolecular correlation effects need to be taken into account. The most efficient approach is to describe the monomers in terms of densities, density matrices, and response properties obtained by density functional theory methods, and the corresponding SAPT variant is termed DFT-SAPT. ${ }^{315-322}$ The DFT-SAPT method was shown to yield very accurate intermolecular interaction energies for small ${ }^{221,318,321,323}$ and large ${ }^{324,325}$ dimer systems. The DFT-SAPT method implemented in the Molpro program can be used with a large variety of different settings in the monomer and the perturbation theory calculations. It should be noted that further approximations are usually employed for exchange contributions: namely, the so-called $S^{2}$ or single-exchange simplified variants of these energies are used, where double and higher simultaneous exchanges of electrons between the monomers are neglected. This approximation works well for the minimum region and for larger distances. However, it can become inaccurate in the valence wall region. Errors in the short-range regions of the intermolecular potential, originating from the common single-exchange approximation to the $E_{\text {exch }}^{(1)}$, 
$E_{\text {exch-ind }}^{(2)}$ and $E_{\text {exch-disp }}^{(2)}$ terms, can be overcome using the infiniteexchange expansion approach derived by Schäffer and Jansen. ${ }^{326,327}$ Charge-transfer interactions, which are a contribution to the sum of $E_{\text {ind }}^{(2)}+E_{\text {exch-ind }}^{(2)}$, can be calculated by the regularized SAPT method of Misquitta. ${ }^{328}$ This variant of the SAPT method can also be employed in cases where the standard perturbative approach breaks down, e.g., in the calculation of heavy metal interactions ${ }^{329}$ or for describing the $\sigma$-hole bond in complexes containing halogen electron donors. $^{330}$

As an alternative to the commonly employed generalized gradient approximations (GGAs) to the exchange-correlation (xc) potential and kernel in DFT-SAPT calculations, it is also possible to use orbital-dependent exact exchange functionals (see Sec. III B) and kernels, ${ }^{331}$ using Molpro. It was recently shown that DFT-SAPT methods which employ the exact nonadiabatic KohnSham exchange kernel (TDEXX) and accurate long-range corrected exchange-correlation potentials in the monomer calculations clearly outperform a large number of empirical and nonempirical quantum chemistry approaches for the popular S22 and S66 dimer benchmark sets of Jurecka et al. ${ }^{209,210,332}$

All contributions in Eq. (8) can be computed using densityfitting techniques in the DFT-SAPT program, leading to remarkable speed-ups in the calculations already for medium sized dimers such as benzene-benzene. ${ }^{221}$ The DFT-SAPT method implemented in the Molpro program was also tested on the S12L set of large supramolecular complexes of Grimme et al. ${ }^{333,334}$ containing dimers with more than 800 electrons. $^{211}$ The interaction energy of the bucky catcher complex (system C4b in the S12L database) can be calculated within 6 days using the DFT-SAPT program on a 16 core Sandy Bridge computer clocked at $2 \mathrm{MHz}$. Here, almost two thirds of the central processing unit (CPU) time were spent in the calculation of the exchange-dispersion energy $E_{\text {exch-disp }}^{(2)}$ that possesses a scaling of $\mathcal{N}^{5}$ with respect to the system size $\mathcal{N}^{211}$ Other interesting applications of this code include endohedral complexes of fullerenes, such as, hydrogen molecule(s) inside $\mathrm{C}_{60}$ and $\mathrm{C}_{70}{ }^{335}$ or $\mathrm{CHFClBr}$ inside chiral isomers of $\mathrm{C}_{82}$.

\section{Symmetry-adapted perturbation theory based on coupled-cluster monomer wavefunctions}

The SAPT with monomers described at the CCSD level has been implemented for all SAPT components of the first and second order with respect to the intermolecular interaction operator [see also Eq. (8)], as described in a number of papers for individual SAPT components. ${ }^{102,337-341}$ It should be noted that dispersion and exchange-dispersion components are calculated from a numerical integration over several imaginary frequencies, where the integrand contains full frequency-dependent polarization propagators at the CCSD level. ${ }^{104}$ Since the calculation of such propagators requires $N^{2}$ responses (for each $\hat{E}_{s}^{r}$ operator), and each of these responses is as expensive as a single CCSD calculation, the cost for full CCSD propagators scales with the eighth power of molecular size $\mathcal{N}$. This scaling can be reduced by one order of $\mathcal{N}$ if density fitting is applied to the electron-density operator entering into the definition of the polarization propagator. ${ }^{340,341}$ Nevertheless, the CCSD-SAPT method is primarily devoted to produce high-quality benchmarks for small noncovalent complexes and to compare them with less accurate methods. For instance, in Ref. 342, the CCSD-SAPT and supermolecular CCSD(T) results were compared to a number of DFT-SAPT approaches with different functionals. The CCSD-SAPT code is also available in the monomercentered basis set (MCBS) or various other subsets of the full dimercentered basis set, thus making use of the SAPT independence on BSSE. It should be noted, however, that the saturation of orbital basis sets for most expensive SAPT components (dispersion and exchange-dispersion) is very slow, which makes the use of the MCBS inefficient in most cases.

\section{USER INTERFACES}

\section{A. Input structure}

The Molpro input is provided in an ASCII file. It contains commands [calling programs as, e.g., $\mathrm{HF}$ and $\operatorname{CCSD}(\mathrm{T})$ ] with associated options and directives which provide additional information such as occupations, symmetry, and spin state. Geometries can be given in xyz or z-matrix form. Any number of commands can follow each other. Branching and looping over parts of the input is also possible. In the beginning of a calculation, the input is pre-checked to detect typos or invalid input specifications at the earliest possible stage. There are facilities to tabulate, plot, or export results, and marked-up xml output is generated as well. Despite the flexibility to generate rather complicated program-like inputs, the input for standard calculations is very simple. Some typical inputs for DFT and coupled-cluster calculations are shown in Table VII.

\section{B. Basis set library}

An extensive basis set library is included in the Molpro program. This includes not only a large variety of orbital basis sets but also a large number of auxiliary basis sets, i.e., basis sets for density fitting in HF, DFT, and correlated methods, as well as sets for approximate identity resolution for explicitly correlated calculations. In addition, a large library of effective core potentials (ECPs) is also maintained that include those from the Los Alamos group, ${ }^{251,252}$ the Stuttgart/Köln group, ${ }^{257,258}$ and others. In particular, the library of orbital basis sets includes all currently available correlation consistent basis sets ${ }^{343}$ across the entire periodic table (see: http://www.grant-hill.group.shef.ac.uk/ccrepo/bib.html). These include relatively new basis sets optimized for scalar relativistic Douglas-Kroll-Hess and X2C Hamiltonians, as well as those matched to Stuttgart/Köln small-core relativistic pseudopotentials, all of which extend the range of this basis set family down to $\mathrm{Z}=103$ (Lr). ${ }^{259,344,345}$ Recent correlation consistent basis sets optimized for explicitly correlated F12 approaches are also included. ${ }^{151-154,346-350}$ Finally, of course, nearly all other commonly used basis sets appearing in the literature (obtained predominately from the basis set exchange at https://www.basissetexchange.org) are also available, including the Karlsruhe def2 sets $^{351,352}$ and Roos atomic natural orbital (ANO) sets. ${ }^{353,354}$ Commonly used auxiliary sets include the JKFIT sets of Weigend, ${ }^{355}$ the MP2FIT series of sets, ${ }^{356-358}$ and the OptRI sets for resolution of the identity in explicitly correlated F12 calculations. ${ }^{359-361}$ The Molpro basis 
TABLE VII. Input examples for a DFT, CCSD(T), and PNO-LCCSD(T) calculation. The auxiliary basis density fitting and RI basis sets are determined automatically.

\begin{tabular}{ll}
\hline $\begin{array}{l}\text { Geometry = mygeom.xyz } \\
\text { Basis = VTZ }\end{array}$ & ! Basis set cc-pVTZ \\
UKS,PBE0,DISP & ! Unrestricted Kohn-Sham with D3 dispersion correction \\
OPTG & ! Geometry optimization \\
Frequencies & ! Harmonic vibrational frequencies \\
\hline Geometry = mygeom.xyz & \\
Basis = VTZ & ! Basis set cc-pVTZ \\
HF & ! Hartree-Fock with density fitting \\
CCSD(T) & ! Conventional coupled-cluster \\
Extrapolate,vtz:vqz:v5z & ! Automatic basis set extrapolation \\
\hline Geometry = mygeom.xyz & \\
Basis = VTZ-F12 & ! Basis set cc-pVTZ-F12 \\
DF-HF & ! Hartree-Fock with density fitting \\
PNO-LCCSD(T)-F12 & ! Explicitly correlated local coupled-cluster \\
\hline \hline
\end{tabular}

set library can be conveniently browsed via the web interface at https://www.molpro.net/info/basis.php.

While the user has complete control over the choices of orbital and auxiliary basis sets for each atom in a given calculation, including any sort of augmentations that might be desired, the program automatically selects appropriate defaults for all required auxiliary basis sets if an orbital basis set from either the correlation consistent or def2 family of basis sets is chosen. This is particularly useful in F12 calculations where three different auxiliary basis sets are required.

In the case of correlation consistent basis sets, Molpro provides a convenient mechanism to perform complete basis set extrapolations. After first specifying the type of energy calculation, the user specifies a range of basis sets, e.g., cc-pVTZ and cc-pVQZ, and then, the required calculations are automatically carried out (cf. Table VII). The user can choose between a large number of common extrapolation formulas, which can be applied separately to reference energies, e.g., HF, and correlation energies. For example, the user can extrapolate the HF energies with an exponential function (requiring three energies) and then the correlation energies with the default $n^{-3}$ formula ( $n$ is the cardinal number of the basis set, e.g., 2 for VDZ and 3 for VTZ). The latter can be carried out with just the two largest basis sets or as a least squares fit to all three basis sets. The results of the extrapolation are automatically saved to variables that can be used in both geometry optimizations and harmonic frequency calculations, as well as output in Molpro's table command.

\section{Graphical user interface: gmolpro}

gmolpro is a GNU Image Manipulation Program Toolkit (GTK)-based graphical interface to Molpro that runs on Linux and macOS workstations. It supports the preparation of inputs through an expert system that interacts with Molpro's registry of commands, methods, and basis sets, guiding the user toward feasible combinations of calculation types (single point, optimization, etc.), methods, basis sets, and options. Checks are implemented so that the geometry, charge, and spin are consistent, and only appropriate methods are selected. Reasonable default settings are provided so that an input can be quickly set up. It also provides a plain-text editor to allow further editing of existing job inputs.

Molecular structures can be constructed and edited using an integrated builder derived from the PQSMol ${ }^{362}$ interface to the $\mathrm{PQS}^{363,364}$ package. It incorporates fragment libraries, force fields, and an optimization based on force fields. Jobs can then be submitted and managed on the local computer or a remote machine (see Sec. VIII D for details). Results may be visualized via the viewer component of PQSMol, including an interactive display of structures, orbitals, property maps, and vibrational modes. Figure 5 shows the PQSMol builder window (upper part) and the gmolpro input window (lower part) for a PNO-LCCSD(T)-F12 calculation on a large transition metal complex.

\section{Job submission and analysis tools}

A new framework for managing individual Molpro runs, collections of calculations, and relationships between them has been developed. The central concept is a project, which is a filesystemresident object, implemented as a directory containing all necessary files, holding everything that belongs to a single run of Molpro. This includes not only the input and output files but also a dictionary of properties which are simple key-value pairs of strings. Some of the property names have special meaning for the operation of the project, but otherwise, anything can be stored in the dictionary. gmolpro makes use of these projects in managing the entire set of data associated with a Molpro calculation but is not the only context in which they can be useful.

The Simple Job Execution Framework (SJEF) ${ }^{365}$ defines a C++ class that provides an interface to these objects; there is also a $\mathrm{C}$ binding and command-line utility, as well a Python library pysjef that includes a binding to SJEF. Although intended for use with Molpro, SJEF has been written in a generic way so that it could be used 


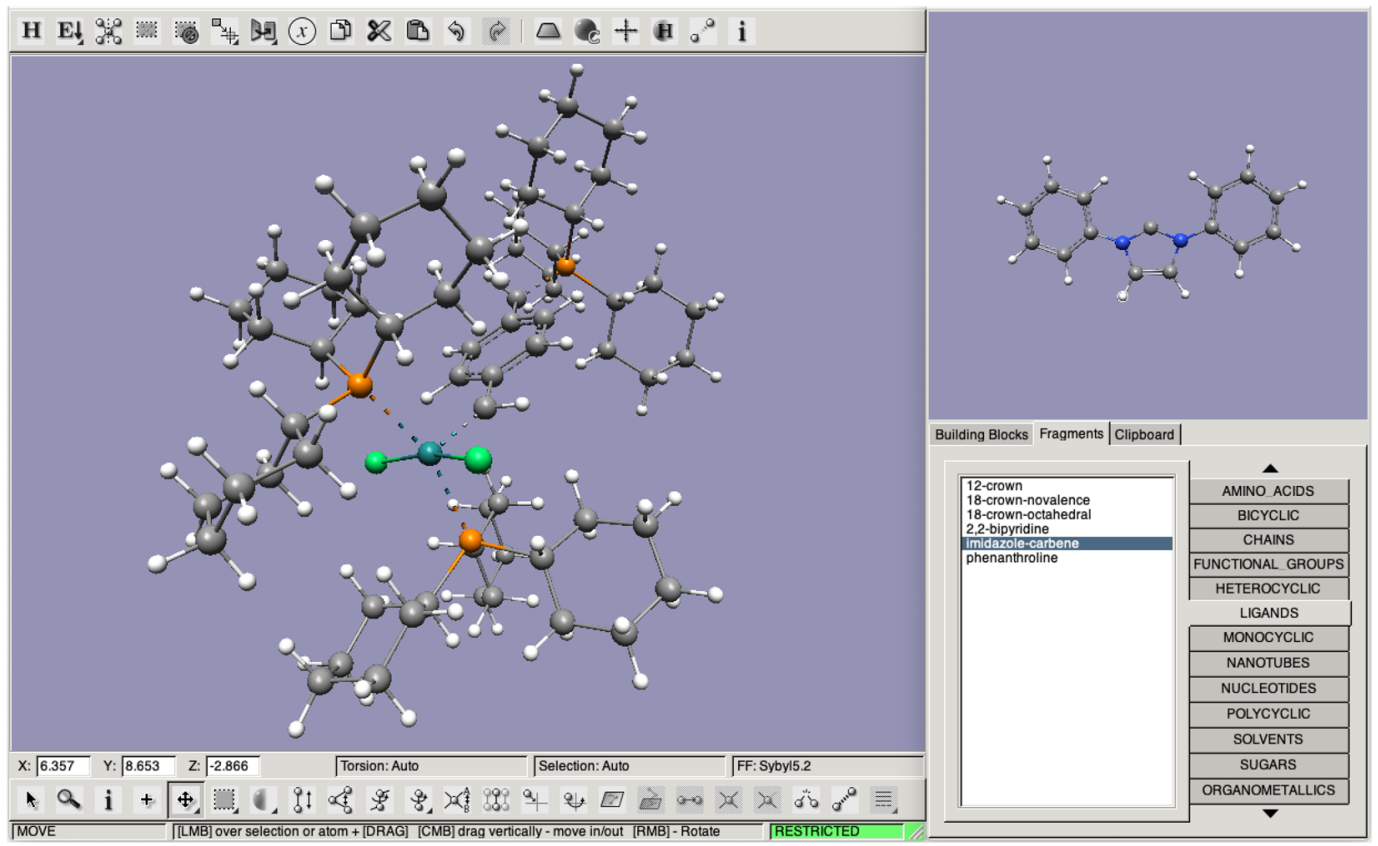

(a)

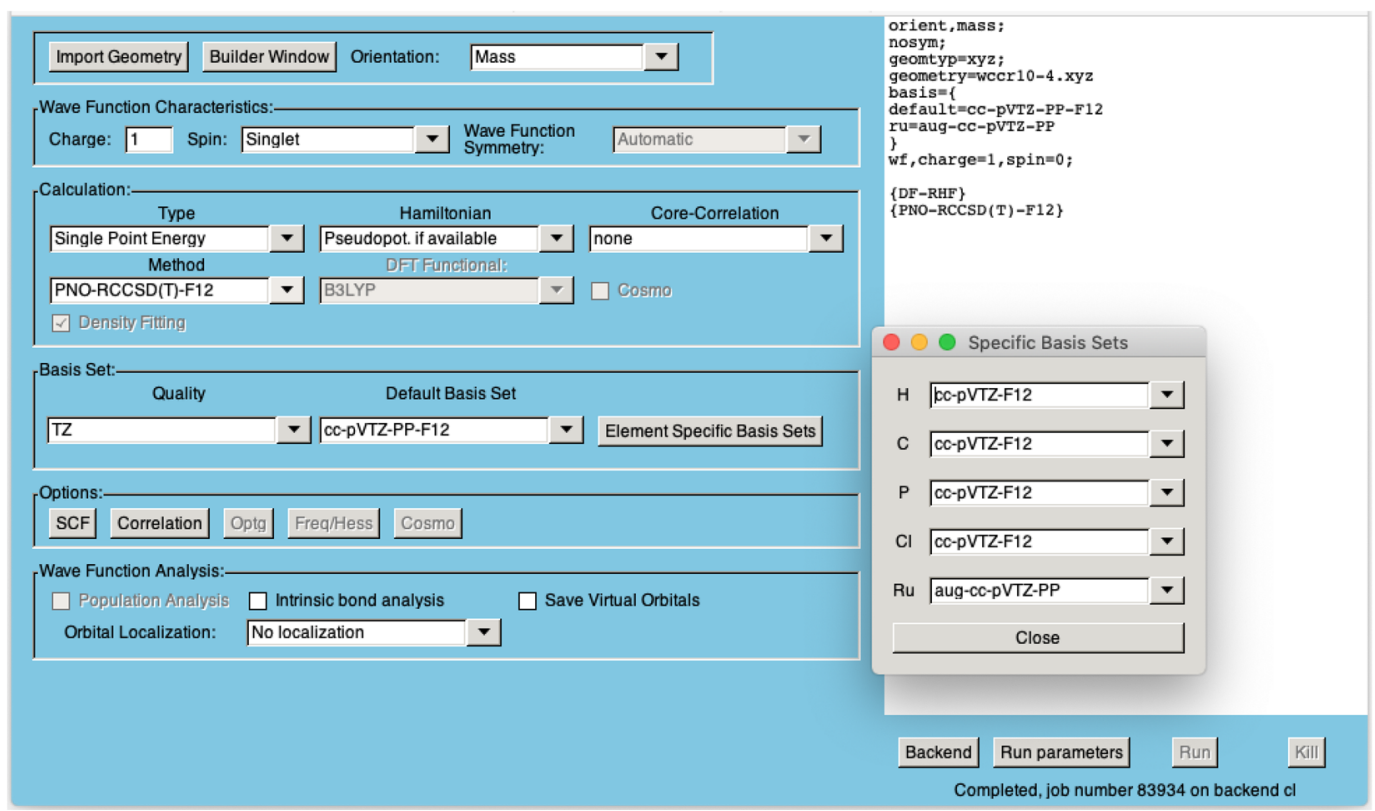

(b)

FIG. 5. (a) gmolpro builder and (b) input and job submission windows. The builder window, based on PQSMol, ${ }^{362}$ shows in its left pane the structure of the reactant molecule of system 4 in the WCCR10 benchmark. ${ }^{195}$ PNO-LCCSD(T)-F12 results for this benchmark can be found in Ref. 56 .

with any other program. As well as providing utilities to manipulate projects and their contents, SJEF supports the launching and monitoring of Molpro runs on either the local computer or a different system. Jobs are placed through the definition of one or more backends that are defined in per-user and per-system configuration files. A cache copy of the project bundle is maintained on the remote host, and synchronization in both directions is carried out as needed. The backend specification contains the command needed to launch 
from Molpro. Programs using this mechanism include $\mathrm{MRCC}^{150}$ and NECI, ${ }^{373}$ and in both cases, the options for the guest code can be specified in the Molpro input.

Conversely, FCIdump files can be read in by Molpro facilitating calculations on model Hamiltonians and frozen virtual approximations, using all available integral-non-direct methods.

\section{B. Development environment, cmake, etc.}

Molpro is written in a mixture of Fortran, $\mathrm{C}$, and $\mathrm{C}++$, and depends on a number of external libraries. In order to manage the complexity of compilation portably, it employs the cmake build system generator. This enables, in particular, the easy incorporation of libraries that themselves have cmake support and the use of an integrated development environment. Most external libraries are specified by reference; if not found locally by cmake, they are fetched and incorporated into the overall build process.

Molpro has a rich interface for basic objects such as orbitals, basis sets, and integral generators, and can be effectively used as a development platform for new quantum-chemical methods.

\section{OUTLOOK AND SUMMARY}

We have described the principal and recent features of the Molpro program package; further details of well-established features can be found in Ref. 31. Many current developments are in progress, including in particular, a strong focus on multireference methodology for large molecules. The package is used by a large community of users and continues to make a distinctive strong contribution to the capability of predicting chemical structure and activity for molecules that are large and that have strongly correlated electronic structure.

\section{ACKNOWLEDGMENTS}

Iakov Polyak acknowledges the support of the Supercomputing Wales project, which is part-funded by the European Regional Development Fund (ERDF) via Welsh Government. Marat Sibaev and Peter Knowles acknowledge the support of EPSRC (Grant No. $\mathrm{EP} / \mathrm{R} 014183 / 1)$.

Molpro has been written by a large community of developers over many years; see https://www.molpro.net/info/authors for a full list. Their work has been made possible by numerous public funders, including the Deutsche Forschungsgemeinschaft (DFG), the European Research Council (ERC Advanced Grant No. 320723, ASES), and the Engineering and Physical Sciences Research Council (EPSRC), as well as Technologie-Transfer-Initiative $\mathrm{GmbH}$ an der Universität Stuttgart (TTI GmbH), the current owner of Molpro. ${ }^{32}$

The data that support the findings of this study are available within the article.

\section{REFERENCES}

${ }^{1}$ W. Meyer, Int. J. Quantum Chem. 5, 341 (1971).

${ }^{2}$ W. Meyer, J. Chem. Phys. 58, 1017 (1973).

${ }^{3}$ P. Pulay, Mol. Phys. 17, 197 (1969).

${ }^{4}$ W. Meyer, Theor. Chim. Acta 35, 277 (1974).

${ }^{5}$ W. Meyer and P. Rosmus, J. Chem. Phys. 63, 2356 (1975).

${ }^{6}$ H.-J. Werner and W. Meyer, Mol. Phys. 31, 855 (1976).

${ }^{7}$ E.-A. Reinsch and W. Meyer, Phys. Rev. A 14, 915 (1976).
${ }^{8}$ P. Rosmus and W. Meyer, J. Chem. Phys. 66, 13 (1977).

${ }^{9}$ P. Rosmus and W. Meyer, J. Chem. Phys. 69, 2745 (1978).

${ }^{10}$ H. -j. Werner and P. J. Knowles, J. Chem. Phys. 89, 5803 (1988).

${ }^{11}$ P. J. Knowles and H.-J. Werner, Chem. Phys. Lett. 145, 514 (1988).

${ }^{12}$ H.-J. Werner and P. J. Knowles, Theor. Chim. Acta 78, 175 (1990).

${ }^{13}$ P. J. Knowles and H.-J. Werner, Theor. Chim. Acta 84, 95 (1992).

${ }^{14}$ K. R. Shamasundar, G. Knizia, and H.-J. Werner, J. Chem. Phys. 135, 054101 (2011).

${ }^{15}$ M. H. Alexander, D. E. Manolopoulos, and H.-J. Werner, J. Chem. Phys. 113, 11084 (2000).

${ }^{16}$ H.-J. Werner, T. B. Adler, and F. R. Manby, J. Chem. Phys. 126, 164102 (2007).

${ }^{17}$ G. Knizia and H.-J. Werner, J. Chem. Phys. 128, 154103 (2008).

${ }^{18}$ T. B. Adler, G. Knizia, and H.-J. Werner, J. Chem. Phys. 127, 221106 (2007).

${ }^{19}$ G. Knizia, T. B. Adler, and H.-J. Werner, J. Chem. Phys. 130, 054104 (2009).

${ }^{20}$ H.-J. Werner, G. Knizia, and F. R. Manby, Mol. Phys. 109, 407 (2011).

${ }^{21}$ T. Shiozaki and H.-J. Werner, J. Chem. Phys. 133, 141103 (2010).

${ }^{22}$ T. Shiozaki, G. Knizia, and H.-J. Werner, J. Chem. Phys. 134, 034113 (2011).

${ }^{23}$ T. Shiozaki and H.-J. Werner, J. Chem. Phys. 134, 184104 (2011).

${ }^{24}$ T. Shiozaki and H.-J. Werner, Mol. Phys. 111, 607 (2013).

${ }^{25}$ D. P. Tew, W. Klopper, C. Neiss, and C. Hättig, Phys. Chem. Chem. Phys. 9, 1921 (2007).

${ }^{26}$ O. Marchetti and H.-J. Werner, Phys. Chem. Chem. Phys. 10, 3400 (2008).

${ }^{27}$ O. Marchetti and H.-J. Werner, J. Phys. Chem. A 113, 11580 (2009).

${ }^{28}$ H.-J. Werner, T. B. Adler, G. Knizia, and F. R. Manby, in Recent Progress in Coupled Cluster Methods, edited by P. Cársky, J. Paldus, and J. Pittner (Springer, Dordrecht; Heidelberg; London; New York, 2010), pp. 573-619.

${ }^{29}$ D. P. Tew, C. Hättig, R. A. Bachorz, and W. Klopper, in Recent Progress in Coupled Cluster Methods, edited by P. Cársky, J. Paldus, and J. Pittner (Springer, Dordrecht; Heidelberg; London; New York, 2010), pp. 535-572.

${ }^{30}$ K. A. Peterson, C. Krause, H. Stoll, J. G. Hill, and H.-J. Werner, Mol. Phys. 109, 2607 (2011).

${ }^{31}$ H.-J. Werner, P. J. Knowles, G. Knizia, F. R. Manby, and M. Schütz, Wiley Interdiscip. Rev.: Comput. Mol. Sci. 2, 242 (2012).

${ }^{32}$ H.-J. Werner, P. J. Knowles, G. Knizia, F. R. Manby, M. Schütz, P. Celani, K. Doll, W. Győrffy, A. Hesselmann, D. Kats, T. Korona, D. Kreplin, R. Lindh, Q. Ma, A. J. May, A. Mitrushenkov, K. A. Peterson, G. Rauhut, K. R. Shamasundar, T. B. Adler, R. D. Amos, S. J. Bennie, A. Bernhardsson, A. Berning, D. L. Cooper, M. J. O. Deegan, A. J. Dobbyn, F. Eckert, E. Goll, C. Hampel, G. Hetzer, T. Hrenar, G. Jansen, C. Köppl, S. J. R. Lee, Y. Liu, A. W. Lloyd, R. A. Mata, S. J. McNicholas, F. Menezes, W. Meyer, T. F. Miller III, M. E. Mura, A. Nicklass, D. P. O’Neill, P. Palmieri, D. Peng, K. Pflüger, R. Pitzer, M. Reiher, M. Schwilk, T. Shiozaki, H. Stoll, A. J. Stone, R. Tarroni, T. Thorsteinsson, D. Usvyat, M. Wang, and M. Welborn, Molpro, version 2020.0, a package of ab initio programs, 2020, see https://www.molpro.net.

${ }^{33}$ D. Kats and F. R. Manby, J. Chem. Phys. 139, 021102 (2013).

${ }^{34}$ D. Kats, J. Chem. Phys. 141, 061101 (2014).

${ }^{35}$ D. Kats, D. Kreplin, H.-J. Werner, and F. R. Manby, J. Chem. Phys. 142, 064111 (2015).

${ }^{36}$ D. Kats, J. Chem. Phys. 144, 044102 (2016).

${ }^{37}$ D. Kats, Mol. Phys. 116, 1435 (2018).

${ }^{38}$ J. B. Robinson and P. J. Knowles, J. Chem. Phys. 136, 054114 (2012).

${ }^{39}$ J. B. Robinson and P. J. Knowles, Phys. Chem. Chem. Phys. 14, 6729 (2012).

${ }^{40}$ J. B. Robinson and P. J. Knowles, J. Chem. Phys. 137, 054301 (2012).

${ }^{41}$ J. B. Robinson and P. J. Knowles, J. Chem. Phys. 138, 074104 (2013).

${ }^{42}$ J. A. Black and P. J. Knowles, Mol. Phys. 116, 1421 (2017).

${ }^{43}$ J. A. Black and P. J. Knowles, J. Chem. Phys. 148, 194102 (2018).

${ }^{44}$ H.-J. Werner, J. Chem. Phys. 129, 101103 (2008).

${ }^{45}$ T. B. Adler and H.-J. Werner, J. Chem. Phys. 130, 241101 (2009).

${ }^{46}$ T. B. Adler, H.-J. Werner, and F. R. Manby, J. Chem. Phys. 130, 054106 (2009).

${ }^{47}$ T. B. Adler and H.-J. Werner, J. Chem. Phys. 135, 144117 (2011).

${ }^{48}$ C. Krause and H.-J. Werner, Phys. Chem. Chem. Phys. 14, 7591 (2012). 
${ }^{49}$ H.-J. Werner, G. Knizia, C. Krause, M. Schwilk, and M. Dornbach, J. Chem. Theory Comput. 11, 484 (2015).

${ }^{50} \mathrm{Q}$. Ma and H.-J. Werner, J. Chem. Theory Comput. 11, 5291 (2015).

${ }^{51}$ H.-J. Werner, C. Köppl, Q. Ma, and M. Schwilk, in Fragmentation: Towards Accurate Calculations on Complex Molecular Systems, edited by M. S. Gordon (Wiley, Chichester, UK, 2017), pp. 1-89, this article is freely available under https://www.wiley.com.

${ }^{52}$ H.-J. Werner, J. Chem. Phys. 145, 201101 (2016).

${ }^{53}$ M. Schwilk, Q. Ma, C. Köppl, and H.-J. Werner, J. Chem. Theory Comput. 13, 3650 (2017).

${ }^{54}$ Q. Ma, M. Schwilk, C. Köppl, and H.-J. Werner, J. Chem. Theory Comput. 13, 4871 (2017).

${ }^{55}$ Q. Ma and H.-J. Werner, J. Chem. Theory Comput. 14, 198 (2017).

${ }^{56}$ Q. Ma and H.-J. Werner, Wiley Interdiscip. Rev.: Comput. Mol. Sci. 8, e1371 (2018).

${ }^{57}$ C. Krause and H.-J. Werner, J. Chem. Theory Comput. 15, 987 (2019).

${ }^{58} \mathrm{Q}$. Ma and H.-J. Werner, "Scalable electron correlation methods. 7. Local open-shell coupled-cluster methods using pair pntural orbitals: PNO-RCCSD and PNO-UCCSD," J. Chem. Theory Comput. (submitted).

${ }^{59}$ F. R. Manby, M. Stella, J. D. Goodpaster, and T. F. Miller III, J. Chem. Theory Comput. 8, 2564 (2012).

${ }^{60}$ D. A. Kreplin, P. J. Knowles, and H.-J. Werner, J. Chem. Phys. 150, 194106 (2019).

${ }^{61}$ D. A. Kreplin, P. J. Knowles, and H.-J. Werner, J. Chem. Phys. 152, 074102 (2020).

${ }^{62}$ F. Menezes, D. Kats, and H.-J. Werner, J. Chem. Phys. 145, 124115 (2016)

${ }^{63}$ D. Kats and H.-J. Werner, J. Chem. Phys. 150, 214107 (2019).

${ }^{64}$ C. Köppl and H.-J. Werner, J. Chem. Theory Comput. 12, 3122 (2016).

${ }^{65}$ P. P. Hallmen, C. Köppl, G. Rauhut, H. Stoll, and J. van Slageren, J. Chem. Phys. 147, 164101 (2017).

${ }^{66}$ P. P. Hallmen, H.-J. Werner, D. Kats, S. Lenz, G. Rauhut, H. Stoll, and J. van Slageren, Phys. Chem. Chem. Phys. 21, 9769 (2019).

${ }^{67}$ L. Roy, M. H. Al-Afyouni, D. E. DeRosha, B. Mondal, I. M. DiMucci, K. M. Lancaster, J. Shearer, E. Bill, W. W. Brennessel, F. Neese, S. Ye, and P. L. Holland, Chem. Sci. 10, 918 (2019).

${ }^{68}$ C. Hampel, K. A. Peterson, and H.-J. Werner, Chem. Phys. Lett. 190, 1 (1992).

${ }^{69} \mathrm{O}$. Christiansen, H. Koch, and P. Jørgensen, Chem. Phys. Lett. 243, 409 (1995).

${ }^{70}$ D. Kats, T. Korona, and M. Schütz, J. Chem. Phys. 125, 104106 (2006).

${ }^{71}$ D. Kats, T. Korona, and M. Schütz, J. Chem. Phys. 127, 064107 (2007).

${ }^{72}$ D. Kats and M. Schütz, J. Chem. Phys. 131, 124117 (2009).

${ }^{73}$ K. Freundorfer, D. Kats, T. Korona, and M. Schütz, J. Chem. Phys. 133, 244110 (2010).

${ }^{74}$ T. Korona and H.-J. Werner, J. Chem. Phys. 118, 3006 (2003).

${ }^{75}$ S. Grimme, J. Chem. Phys. 118, 9095 (2003).

${ }^{76}$ P. J. Knowles, C. Hampel, and H.-J. Werner, J. Chem. Phys. 99, 5219 (1993).

${ }^{77}$ D. Kats and D. P. Tew, J. Chem. Theory Comput. 15, 13 (2019).

${ }^{78}$ M. Schütz, R. Lindh, and H.-J. Werner, Mol. Phys. 96, 719 (1999).

${ }^{79}$ B. Cooper and P. J. Knowles, J. Chem. Phys. 133, 234102 (2010).

${ }^{80} \mathrm{P}$. Piecuch, S. A. Kucharski, and K. Kowalski, Chem. Phys. Lett. 344, 176 (2001).

${ }^{81}$ T. Van Voorhis and M. Head-Gordon, J. Chem. Phys. 113, 8873 (2000).

${ }^{82}$ P. Piecuch and M. Włoch, J. Chem. Phys. 123, 224105 (2005).

${ }^{83}$ A. I. Krylov, L. V. Slipchenko, and S. V. Levchenko, Electron Correlation Methodology, ACS Symposium Series Vol. 958 (American Chemical Society, 2007), pp. 89-102.

${ }^{84}$ D. Kats and A. Köhn, J. Chem. Phys. 150, 151101 (2019).

${ }^{85}$ J. F. Stanton and R. J. Bartlett, J. Chem. Phys. 98, 7029 (1993).

${ }^{86}$ E. R. Davidson, J. Comput. Phys. 17, 87 (1975).

${ }^{87}$ K. Hirao and H. Nakatsuji, J. Comput. Phys. 45, 246 (1982).

${ }^{88}$ J. F. Stanton and J. Gauss, J. Chem. Phys. 111, 8785 (1999).

${ }^{89}$ T. Korona, Mol. Phys. 110, 199 (2012).

${ }^{90}$ F. Plasser and H. Lischka, J. Chem. Theory Comput. 8, 2777 (2012).

${ }^{91}$ G. Wälz, D. Kats, D. Usvyat, T. Korona, and M. Schütz, Phys. Rev. A 86, 052519 (2012).
${ }^{92}$ D. M. P. Holland, A. B. Trofimov, E. A. Seddon, E. V. Gromov, T. Korona, N. de Oliveira, L. E. Archer, D. Joyeux, and L. Nahon, Phys. Chem. Chem. Phys. 16, 21629 (2014).

${ }^{93}$ D. M. P. Holland, E. A. Seddon, A. B. Trofimov, E. V. Gromov, M. Wormit, A. Dreuw, T. Korona, N. De Oliveira, L. E. Archer, and D. Joyeux, J. Mol, Spectrosc. 315, 184 (2015).

${ }^{94}$ D. Rutkowska-Zbik and T. Korona, J. Chem. Theory Comput. 8, 2972 (2012).

${ }^{95}$ A. C. Scheiner, G. E. Scuseria, J. E. Rice, T. J. Lee, and H. F. Schaefer, J. Chem. Phys. 87, 5361 (1987).

${ }^{96}$ P. Jørgensen and T. Helgaker, J. Chem. Phys. 89, 1560 (1988).

${ }^{97}$ H. Koch, H. J. A. Jensen, P. Jørgensen, T. Helgaker, G. E. Scuseria, and H. F. Schaefer, J. Chem. Phys. 92, 4924 (1990).

${ }^{98}$ T. Korona, R. Moszynski, and B. Jeziorski, Mol. Phys. 100, 1723 (2002).

${ }^{99}$ G. Rauhut and H.-J. Werner, Phys. Chem. Chem. Phys. 3, 4853 (2001).

${ }^{100}$ R. J. Bartlett and J. Noga, Chem. Phys. Lett. 150, 29 (1988).

${ }^{101}$ B. Jeziorski and R. Moszynski, Int. J. Quantum Chem. 48, 161 (1993).

${ }^{102}$ T. Korona, J. Chem. Phys. 128, 224104 (2008).

${ }^{103}$ R. Moszynski, P. S. Żuchowski, and B. Jeziorski, Collect. Czech. Chem. Commun. 70, 1109 (2005).

${ }^{104}$ T. Korona, M. Przybytek, and B. Jeziorski, Mol. Phys. 104, 2303 (2006).

${ }^{105}$ T. Korona, Mol. Phys. 108, 343 (2010).

${ }^{106}$ H.-J. Werner and W. Meyer, J. Chem. Phys. 73, 2342 (1980).

${ }^{107}$ H.-J. Werner and W. Meyer, J. Chem. Phys. 74, 5794 (1981).

${ }^{108}$ H.-J. Werner and P. J. Knowles, J. Chem. Phys. 82, 5053 (1985).

${ }^{109}$ P. J. Knowles and H.-J. Werner, Chem. Phys. Lett. 115, 259 (1985).

${ }^{110}$ P. J. Knowles and N. C. Handy, Chem. Phys. Lett. 111, 315 (1984).

${ }^{111}$ P. A. Malmqvist, A. Rendell, and B. O. Roos, J. Phys. Chem. 94, 5477 (1990).

${ }^{112}$ B. O. Roos, Int. J. Quantum Chem. 18, 175 (1980).

113 J. Nocedal, Math. Comput. 35, 773 (1980).

${ }^{114}$ E. R. Sayfutyarova, Q. Sun, G. K.-L. Chan, and G. Knizia, J. Chem. Theory Comput. 13, 4063 (2017).

${ }^{115}$ Y. Guo, K. Sivalingam, E. F. Valeev, and F. Neese, J. Chem. Phys. 144, 094111 (2016).

${ }^{116}$ W. Meyer, "Configuration expansion by means of pseudonatural orbitals," in Methods of Electronic Structure Theory, Modern Theoretical Chemistry Vol. 3, edited by H. F. Schaefer III (Plenum, New York, 1977).

${ }^{117}$ P. E. M. Siegbahn, Int. J. Quantum Chem. 18, 1229 (1980).

${ }^{118}$ P. E. M. Siegbahn and M. Svensson, Int. J. Quantum Chem. 41, 153 (1992).

${ }^{119}$ H.-J. Werner and E.-A. Reinsch, in Proceedings of the Fifth Seminar on Computational Methods in Quantum Chemistry (Max-Planck-Institut für Physik und Astrophysik, München; Groningen, The Netherlands, 1981).

${ }^{120}$ H.-J. Werner and E. A. Reinsch, J. Chem. Phys. 76, 3144 (1982).

${ }^{121}$ R. J. Gdanitz and R. Ahlrichs, Chem. Phys. Lett. 143, 413 (1988).

${ }^{122}$ R. J. Cave and E. R. Davidson, J. Chem. Phys. 88, 5770 (1988).

${ }^{123}$ R. J. Cave and E. R. Davidson, J. Chem. Phys. 89, 6798 (1988).

${ }^{124}$ P. Celani and H.-J. Werner, J. Chem. Phys. 112, 5546 (2000).

${ }^{125}$ D. Kats and F. R. Manby, J. Chem. Phys. 138, 144101 (2013).

${ }^{126}$ W. Győrffy, G. Knizia, and H.-J. Werner, J. Chem. Phys. 147, 214101 (2017).

${ }^{127}$ W. Györffy and H.-J. Werner, J. Chem. Phys. 148, 114104 (2018).

${ }^{128}$ H.-J. Werner, Mol. Phys. 89, 645 (1996).

${ }^{129}$ T. Shiozaki, W. Györffy, P. Celani, and H.-J. Werner, J. Chem. Phys. 135, 081106 (2011).

${ }^{130}$ T. Shiozaki, C. Woywod, and H.-J. Werner, Phys. Chem. Chem. Phys. 15, 262 (2013).

${ }^{131}$ A. A. Granovsky, J. Chem. Phys. 134, 214113 (2011).

${ }^{132}$ D. I. Lyakh, M. Musiał, V. F. Lotrich, and R. J. Bartlett, Chem. Rev. 112, 182 (2012).

${ }^{133}$ A. Köhn, M. Hanauer, L. A. Mück, T.-C. Jagau, and J. Gauss, Wiley Interdiscip. Rev.: Comput. Mol. Sci. 3, 176 (2013).

${ }^{134}$ F. A. Evangelista, J. Chem. Phys. 149, 030901 (2018).

${ }^{135}$ A. Banerjee and J. Simons, Int. J. Quantum Chem. 19, 207 (1981).

${ }^{136}$ A. Banerjee and J. Simons, J. Chem. Phys. 76, 4548 (1982). 
${ }^{137}$ F. A. Evangelista and J. Gauss, J. Chem. Phys. 134, 114102 (2011).

${ }^{138}$ M. Hanauer and A. Köhn, J. Chem. Phys. 134, 204111 (2011).

${ }^{139}$ M. Hanauer and A. Köhn, J. Chem. Phys. 136, 204107 (2012).

${ }^{140}$ M. Hanauer and A. Köhn, J. Chem. Phys. 137, 131103 (2012).

${ }^{141}$ Y. A. Aoto and A. Köhn, J. Chem. Phys. 144, 074103 (2016).

${ }^{142}$ Y. A. Aoto and A. Köhn, Phys. Chem. Chem. Phys. 18, 30241 (2016).

${ }^{143}$ Y. A. Aoto, A. P. de Lima Batista, A. Köhn, and A. G. S. de Oliveira-Filho, J. Chem. Theory Comput. 13, 5291 (2017).

${ }^{144}$ D. J. Coughtrie, R. Giereth, D. Kats, H.-J. Werner, and A. Köhn, J. Chem. Theory Comput. 14, 693 (2018).

${ }^{145}$ R. A. Mata, H.-J. Werner, and M. Schütz, J. Chem. Phys. 128, 144106 (2008).

${ }^{146}$ G. Knizia, J. Chem. Theory Comput. 9, 4834 (2013).

${ }^{147}$ Y. A. Aoto, A. Bargholz, D. Kats, H.-J. Werner, and A. Köhn, J. Chem. Theory Comput. 15, 2291 (2019).

${ }^{148}$ J. A. Black and A. Köhn, J. Chem. Phys. 150, 194107 (2019).

${ }^{149}$ A. Köhn and A. Bargholz, J. Chem. Phys. 151, 041106 (2019).

${ }^{150}$ MRCC, a quantum chemical program suite written by M. Kállay, P. R. Nagy, Z. Rolik, D. Mester, G. Samu, J. Csontos, J. Csóka, B. P. Szabó, L. Gyevi-Nagy, I. Ladjánszki, L. Szegedy, B. Ladóczki, K. Petrov, M. Farkas, P. D. Mezei, and B. Hégely. See also Z. Rolik, L. Szegedy, I. Ladjánszki, B. Ladóczki, and M. Kállay, J. Chem. Phys. 139, 094105 (2013), as well as www.mrcc.hu.

${ }^{151}$ K. A. Peterson, T. B. Adler, and H.-J. Werner, J. Chem. Phys. 128, 084102 (2008).

${ }^{152}$ J. G. Hill, S. Mazumder, and K. A. Peterson, J. Chem. Phys. 132, 054108 (2010).

${ }^{153}$ D. H. Bross, J. G. Hill, H.-J. Werner, and K. A. Peterson, J. Chem. Phys. 139, 094302 (2013).

${ }^{154}$ J. G. Hill and K. A. Peterson, J. Chem. Phys. 141, 094106 (2014).

${ }^{155}$ H.-J. Werner, G. Knizia, T. B. Adler, and O. Marchetti, Z. Phys. Chem. 224, 493 (2010).

${ }^{156}$ D. Feller, K. A. Peterson, and J. G. Hill, J. Chem. Phys. 133, 184102 (2010).

${ }^{157}$ J. G. Hill, K. A. Peterson, G. Knizia, and H.-J. Werner, J. Chem. Phys. 131, 194105 (2009).

${ }^{158}$ J. G. Hill and K. A. Peterson, J. Chem. Theor. Comput. 8, 518 (2012).

${ }^{159}$ Q. Ma and H.-J. Werner, J. Chem. Theory Comput. 15, 1044 (2019).

${ }^{160}$ N. Sylvetsky, M. K. Kesharwani, and J. M. L. Martin, J. Chem. Phys. 147, 134106 (2017).

${ }^{161}$ J. M. Foster and S. F. Boys, Rev. Mod. Phys. 32, 300 (1960).

${ }^{162}$ J. Pipek and P. G. Mezey, J. Chem. Phys. 90, 4916 (1989).

${ }^{163}$ W. C. Lu, C. Z. Wang, M. W. Schmidt, L. Bytautas, K. M. Ho, and K. Ruedenberg, J. Chem. Phys. 120, 2629 (2004).

${ }^{164}$ C. Hampel and H.-J. Werner, J. Chem. Phys. 104, 6286 (1996).

${ }^{165}$ G. Hetzer, P. Pulay, and H.-J. Werner, Chem. Phys. Lett. 290, 143 (1998).

${ }^{166}$ M. Schütz, G. Hetzer, and H.-J. Werner, J. Chem. Phys. 111, 5691 (1999).

${ }^{167}$ G. Hetzer, M. Schütz, H. Stoll, and H.-J. Werner, J. Chem. Phys. 113, 9443 (2000).

${ }^{168}$ J. Gauss and H.-J. Werner, Phys. Chem. Chem. Phys. 2, 2083 (2000).

${ }^{169}$ M. Schütz and H.-J. Werner, Chem. Phys. Lett. 318, 370 (2000).

${ }^{170}$ M. Schütz, J. Chem. Phys. 113, 9986 (2000).

${ }^{171}$ M. Schütz and H.-J. Werner, J. Chem. Phys. 114, 661 (2001).

${ }^{172}$ M. Schütz, J. Chem. Phys. 116, 8772 (2002).

${ }^{173}$ M. Schütz, Phys. Chem. Chem. Phys. 4, 3941 (2002).

${ }^{174}$ H.-J. Werner, F. R. Manby, and P. J. Knowles, J. Chem. Phys. 118, 8149 (2003).

${ }^{175}$ M. Schütz, H.-J. Werner, R. Lindh, and F. R. Manby, J. Chem. Phys. 121, 737 (2004).

${ }^{176}$ R. A. Mata and H.-J. Werner, J. Chem. Phys. 125, 184110 (2006).

${ }^{177}$ H.-J. Werner and K. Pflüger, Annu. Rep. Comput. Chem. 2, 53 (2006).

${ }^{178}$ R. A. Mata and H.-J. Werner, Mol. Phys. 105, 2753 (2007).

${ }^{179}$ T. Hrenar, G. Rauhut, and H.-J. Werner, J. Phys. Chem. A 110, 2060 (2006).

${ }^{180}$ H.-J. Werner and M. Schütz, J. Chem. Phys. 135, 144116 (2011).

${ }^{181}$ A. El Azhary, G. Rauhut, P. Pulay, and H.-J. Werner, J. Chem. Phys. 108, 5185 (1998).

${ }^{182}$ M. Dornbach and H.-J. Werner, Mol. Phys. 117, 1252 (2018).
${ }^{183}$ K. Ledermüller, D. Kats, and M. Schütz, J. Chem. Phys. 139, 084111 (2013).

${ }^{184}$ K. Ledermüller and M. Schütz, J. Chem. Phys. 140, 164113 (2014).

${ }^{185}$ J. Yang, Y. Kurashige, F. R. Manby, and G. K. L. Chan, J. Chem. Phys. 134, 044123 (2011).

${ }^{186}$ J. Yang, G. K.-L. Chan, F. R. Manby, M. Schütz, and H.-J. Werner, J. Chem. Phys. 136, 144105 (2012).

${ }^{187}$ M. Schütz, J. Yang, G. K.-L. Chan, F. R. Manby, and H.-J. Werner, J. Chem. Phys. 138, 054109 (2013).

${ }^{188}$ M. Schwilk, D. Usvyat, and H.-J. Werner, J. Chem. Phys. 142, 121102 (2015).

${ }^{189}$ J. Nieplocha, B. Palmer, V. Tipparaju, M. Krishnan, H. Trease, and E. Aprà, Int. J. High Perform. Comput. Appl. 20, 203 (2006).

${ }^{190} \mathrm{~J}$. Friedrich and J. Hänchen, J. Chem. Theory Comput. 9, 5381 (2013).

${ }^{191}$ M. K. Kesharwani, A. Karton, N. Sylvetsky, and J. M. L. Martin, Aust. J. Chem. 71, 238 (2018).

${ }^{192}$ Y. Liu, "Linear scaling high-spin open-shell local correlation methods," Ph.D. thesis, University of Stuttgart, 2011.

${ }^{193}$ S. Luo, Y. Zhao, and D. G. Truhlar, Phys. Chem. Chem. Phys. 13, 13683 (2011).

${ }^{194}$ A. Fedorov, L. Batiste, E. P. A. Couzijn, and P. Chen, ChemPhysChem 11, 1002 (2010).

${ }^{195}$ T. Weymuth, E. P. A. Couzijn, P. Chen, and M. Reiher, J. Chem. Theory Comput. 10, 3092 (2014).

${ }^{196}$ M. Radoń, Phys. Chem. Chem. Phys. 21, 4854 (2019).

${ }^{197}$ D. Kats, J. Chem. Phys. 141, 244101 (2014).

${ }^{198}$ D. Kats, J. Chem. Phys. 145, 014103 (2016).

${ }^{199}$ S. Grimme, J. Antony, S. Ehrlich, and H. Krieg, J. Chem. Phys. 132, 154104 (2010).

${ }^{200}$ E. Caldeweyher, C. Bannwarth, and S. Grimme, J. Chem. Phys. 147, 034112 (2017).

${ }^{201}$ A. Heßelmann, J. Chem. Theory Comput. 9, 273 (2013).

${ }^{202}$ A. Heßelmann, J. Chem. Phys. 149, 044103 (2018).

${ }^{203}$ F. Della Sala and A. Görling, J. Chem. Phys. 115, 5718 (2001).

${ }^{204}$ A. Heßelmann, A. W. Götz, F. Della Sala, and A. Görling, J. Chem. Phys. 127, 054102 (2007).

${ }^{205}$ A. D. Becke, J. Chem. Phys. 88, 2547 (1988).

${ }^{206}$ C. W. Murray, N. C. Handy, and G. J. Laming, Mol. Phys. 78, 997 (1993).

${ }^{207}$ R. Strange, F. R. Manby, and P. J. Knowles, Comput. Phys. Commun. 136, 310 (2001).

${ }^{208}$ S. Grimme, A. Hansen, J. G. Brandenburg, and C. Bannwarth, Chem. Rev. 116, 5105 (2016).

${ }^{209}$ P. Jurecka, J. Sponer, J. Cerny, and P. Hobza, Phys. Chem. Chem. Phys. 8, 1985 (2006).

${ }^{210}$ J. Rezac, K. E. Riley, and P. Hobza, J. Chem. Theory Comput. 10, 1359 (2014).

${ }^{211}$ A. Heßelmann and T. Korona, J. Chem. Phys. 141, 094107 (2014).

${ }^{212}$ A. Holas and M. Cinal, Phys. Rev. A 72, 032504 (2005).

${ }^{213}$ A. Görling, J. Chem. Phys. 123, 062203 (2005).

${ }^{214}$ A. Heßelmann and A. Görling, J. Chem. Phys. 134, 034120 (2011).

${ }^{215}$ R. T. Sharp and G. K. Horton, Phys. Rev. 90, 317 (1953).

${ }^{216}$ J. D. Talman and W. F. Shadwick, Phys. Rev. A 14, 36 (1976).

${ }^{217}$ A. Görling, Phys. Rev. Lett. 83, 5459 (1999).

${ }^{218}$ S. Hamel, M. E. Casida, and D. R. Salahub, J. Chem. Phys. 116, 8276 (2002).

${ }^{219}$ M. Grüning, O. V. Gritsenko, and E. J. Baerends, J. Chem. Phys. 116, 6435 (2002).

${ }^{220}$ V. N. Staroverov, G. E. Scuseria, and E. R. Davidson, J. Chem. Phys. 125, 081104 (2006).

${ }^{221}$ A. Heßelmann, G. Jansen, and M. Schütz, J. Chem. Phys. 122, 014103 (2005).

${ }^{222}$ Time-Dependent Density Functional Theory, Lecture Notes in Physics Vol. 706, edited by M. A. L. Marques, C. A. Ullrich, F. Nogueira, A. Rubio, K. Burke, and E. K. U. Gross (Springer, Heidelberg, 2006).

${ }^{223}$ A. Heßelmann, A. Ipatov, and A. Görling, Phys. Rev. A 80, 012507 (2009).

${ }^{224}$ A. Görling, A. Ipatov, A. W. Götz, and A. Heßelmann, Z. Phys. Chem. 224, 325 (2010).

${ }^{225}$ A. Ipatov, A. Heßelmann, and A. Görling, Int. J. Quantum Chem. 110, 2202 (2010). 
${ }^{226}$ P. Sherwood, A. H. de Vries, M. F. Guest, G. Schreckenbach, C. R. A. Catlow, S. A. French, A. A. Sokol, S. T. Bromley, W. Thiel, A. J. Turner et al., J. Mol. Struct.: THEOCHEM 632, 1 (2003).

${ }^{227}$ G. R. Ahmadi and J. Almlöf, Chem. Phys. Lett. 246, 364 (1995).

${ }^{228}$ C. A. White and M. Head-Gordon, J. Chem. Phys. 104, 2620 (1996).

${ }^{229}$ F. Claeyssens, J. N. Harvey, F. R. Manby, R. A. Mata, A. J. Mulholland, K. E. Ranaghan, M. Schütz, S. Thiel, W. Thiel, and H.-J. Werner, Angew. Chem., Int. Ed. 45, 6856 (2006).

${ }^{230}$ R. A. Mata, H.-J. Werner, S. Thiel, and W. Thiel, J. Chem. Phys. 128, 025104 (2008).

${ }^{231}$ M. W. van der Kamp, J. Żurek, F. R. Manby, J. N. Harvey, and A. J. Mulholland, J. Phys. Chem. B 114, 11303 (2010).

${ }^{232}$ N. Lawan, K. E. Ranaghan, F. R. Manby, and A. J. Mulholland, Chem. Phys. Lett. 608, 380 (2014).

${ }^{233}$ M. Stella, S. J. Bennie, and F. R. Manby, Mol. Phys. 113, 1858 (2015).

${ }^{234}$ T. A. Barnes, J. W. Kaminski, O. Borodin, and T. F. Miller III, J. Phys. Chem. C 119, 3865 (2015).

${ }^{235}$ P. Huo, C. Uyeda, J. D. Goodpaster, J. C. Peters, and T. F. Miller III, ACS Catal. 6, 6114 (2016)

${ }^{236}$ S. J. Bennie, M. W. van der Kamp, R. C. R. Pennifold, M. Stella, F. R. Manby, and A. J. Mulholland, J. Chem. Theory Comput. 12, 2689 (2016).

${ }^{237}$ S. J. Bennie, B. F. E. Curchod, F. R. Manby, and D. R. Glowacki, J. Phys. Chem. Lett. 8, 5559 (2017).

${ }^{238}$ M. Welborn, F. R. Manby, and T. F. Miller III, J. Chem. Phys. 149, 144101 (2018).

${ }^{239}$ X. Zhang, S. J. Bennie, M. W. van der Kamp, D. R. Glowacki, F. R. Manby, and A. J. Mulholland, R. Soc. Open Sci. 5, 171390 (2018).

${ }^{240}$ A. Chapovetsky, M. Welborn, J. M. Luna, R. Haiges, T. F. Miller III, and S. C. Marinescu, ACS Cent. Sci. 4, 397 (2018).

${ }^{241}$ S. J. Bennie, M. Stella, T. F. Miller III, and F. R. Manby, J. Chem. Phys. 143, 024105 (2015).

${ }^{242}$ S. J. R. Lee, F. Ding, F. R. Manby, and T. F. Miller III, J. Chem. Phys. 151, 064112 (2019).

${ }^{243}$ M. Douglas and N. M. Kroll, Ann. Phys. 82, 89 (1974).

${ }^{244}$ B. A. Hess, Phys. Rev. A 33, 3742 (1986).

${ }^{245}$ G. Jansen and B. A. Hess, Phys. Rev. A 39, 6016 (1989).

${ }^{246}$ M. Iliaš and T. Saue, J. Chem. Phys. 126, 004102 (2007).

${ }^{247}$ D. Peng and M. Reiher, Theor. Chem. Acc. 131, 1081 (2012).

${ }^{248} \mathrm{M}$. Reiher and A. Wolf, J. Chem. Phys. 121, 10945 (2004).

${ }^{249}$ L. Visscher and K. G. Dyall, At. Data Nucl. Data Tables 67, 207 (1997).

${ }^{250}$ M. Dolg and X. Cao, Chem. Rev. 112, 403 (2012).

${ }^{251}$ W. R. Wadt and P. J. Hay, J. Chem. Phys. 82, 284 (1985).

${ }^{252}$ P. J. Hay and W. R. Wadt, J. Chem. Phys. 82, 270 (1985).

${ }^{253}$ W. J. Stevens, H. Basch, and M. Krauss, J. Chem. Phys. 81, 6026 (1984).

${ }^{254}$ W. J. Stevens, M. Krauss, H. Basch, and P. G. Jasien, Can. J. Chem. 70, 612 (1992).

${ }^{255}$ J. R. Trail and R. J. Needs, J. Chem. Phys. 122, 174109 (2005).

${ }^{256}$ J. R. Trail and R. J. Needs, J. Chem. Phys. 142, 064110 (2015).

${ }^{257}$ H. Stoll, B. Metz, and M. Dolg, J. Comput. Chem. 23, 767 (2002).

${ }^{258}$ A. Weigand, X. Cao, T. Hangele, and M. Dolg, J. Phys. Chem. A 118, 2519 (2014).

${ }^{259}$ D. Figgen, K. A. Peterson, M. Dolg, and H. Stoll, J. Chem. Phys. 130, 164108 (2009).

${ }^{260}$ B. A. Heß, C. M. Marian, U. Wahlgren, and O. Gropen, Chem. Phys. Lett. 251, 365 (1996).

${ }^{261}$ A. Berning, M. Schweizer, H.-J. Werner, P. J. Knowles, and P. Palmieri, Mol. Phys. 98, 1823 (2000).

${ }^{262}$ P. Celani and H.-J. Werner, J. Chem. Phys. 119, 5044 (2003).

${ }^{263}$ W. Györffy, T. Shiozaki, G. Knizia, and H.-J. Werner, J. Chem. Phys. 138, 104104 (2013)

${ }^{264}$ F. Eckert, P. Pulay, and H.-J. Werner, J. Comput. Chem. 18, 1473 (1997).

${ }^{265}$ F. Eckert and H.-J. Werner, Theor. Chem. Acc. 100, 21 (1998).
${ }^{266}$ J. K. G. Watson, Mol. Phys. 15, 479 (1968).

${ }^{267}$ G. Rauhut, J. Chem. Phys. 121, 9313 (2004).

${ }^{268}$ B. Ziegler and G. Rauhut, J. Chem. Phys. 149, 164110 (2018).

${ }^{269}$ D. Oschetzki, M. Neff, P. Meier, F. Pfeiffer, and G. Rauhut, Croat. Chem. Acta 85, 379 (2012).

${ }^{270}$ J. M. Bowman, T. Carrington, Jr., and H.-D. Meyer, Mol. Phys. 106, 2145 (2008).

${ }^{271}$ B. Ziegler and G. Rauhut, J. Chem. Theory Comput. 15, 4187 (2019).

${ }^{272}$ K. Pflüger, M. Paulus, S. Jagiella, T. Burkert, and G. Rauhut, Theor. Chem. Acc. 114, 327 (2005)

${ }^{273}$ K. Yagi, S. Hirata, and K. Hirao, Theor. Chem. Acc. 118, 681 (2007).

${ }^{274} \mathrm{M}$. Neff and G. Rauhut, Spectrochim. Acta, Part A 119, 100 (2014).

${ }^{275}$ G. Rauhut and B. Hartke, J. Chem. Phys. 131, 014108 (2009).

${ }^{276}$ P. Meier, G. Bellchambers, J. Klepp, F. R. Manby, and G. Rauhut, Phys. Chem. Chem. Phys. 15, 10233 (2013).

${ }^{277}$ B. Ziegler and G. Rauhut, J. Chem. Phys. 144, 114114 (2016).

${ }^{278}$ J. Meisner, P. P. Hallmen, J. Kästner, and G. Rauhut, J. Chem. Phys. 150, 084306 (2019).

${ }^{279}$ S. Heislbetz and G. Rauhut, J. Chem. Phys. 132, 124102 (2010).

${ }^{280}$ S. Heislbetz, F. Pfeiffer, and G. Rauhut, J. Chem. Phys. 134, 204108 (2011).

${ }^{281}$ F. Pfeiffer and G. Rauhut, J. Phys. Chem. A 115, 11050 (2011).

${ }^{282}$ M. Neff, T. Hrenar, D. Oschetzki, and G. Rauhut, J. Chem. Phys. 134, 064105 (2011).

${ }^{283}$ M. Neff and G. Rauhut, J. Chem. Phys. 131, 124129 (2009).

${ }^{284}$ F. Pfeiffer and G. Rauhut, J. Chem. Phys. 140, 064110 (2014).

${ }^{285}$ P. Meier, D. Oschetzki, F. Pfeiffer, and G. Rauhut, J. Chem. Phys. 143, 24411 (2015).

${ }^{286}$ D. Oschetzki and G. Rauhut, Phys. Chem. Chem. Phys. 16, 16426 (2014).

${ }^{287}$ T. Petrenko and G. Rauhut, J. Chem. Phys. 146, 124101 (2017).

${ }^{288}$ X. Zhao, X. Chu, G. Rauhut, C. Chen, C. Song, B. Lu, and X. Zeng, Angew. Chem., Int. Ed. 58, 12164 (2019).

${ }^{289}$ B. Ziegler and G. Rauhut, Mol. Phys. 117, 1741 (2019).

${ }^{290}$ R. Ramakrishnan and G. Rauhut, J. Chem. Phys. 142, 154118 (2015).

${ }^{291}$ P. Meier and G. Rauhut, Mol. Phys. 113, 3859 (2015).

${ }^{292}$ P. Meier, D. Oschetzki, R. Berger, and G. Rauhut, J. Chem. Phys. 140, 184111 (2014).

${ }^{293}$ G. Rauhut, J. Phys. Chem. A 119, 10264 (2015).

${ }^{294}$ T. Petrenko and G. Rauhut, J. Chem. Phys. 143, 234106 (2015).

${ }^{295}$ T. Petrenko and G. Rauhut, J. Chem. Theory Comput. 13, 5515 (2017).

${ }^{296}$ A. Mitrushchenkov and H.-J. Werner, Mol. Phys. 105, 1239 (2007).

${ }^{297}$ A. J. Stone, Chem. Phys. Lett. 83, 233 (1981).

${ }^{298}$ J. E. M. N. Klein, G. Knizia, B. Miehlich, J. Kästner, and B. Plietker, Chem. - Eur. J. 20, 7254 (2014).

${ }^{299}$ G. Knizia and J. E. M. N. Klein, Angew. Chem., Int. Ed. 54, 5518 (2015).

${ }^{300}$ L. N. dos Santos Comprido, J. E. M. N. Klein, G. Knizia, J. Kästner, and A. S. Hashmi, Angew. Chem., Int. Ed. 54, 10336 (2015).

${ }^{301}$ J. E. M. N. Klein, G. Knizia, L. Nunes Dos Santos Comprido, J. Kästner, and A. S. K. Hashmi, Chem. - Eur. J. 23, 16097 (2017).

${ }^{302}$ J. S. Steen, G. Knizia, and J. E. M. N. Klein, Angew. Chem. 131, 13267 (2019).

${ }^{303}$ E. D. Glendening, C. R. Landis, and F. Weinhold, J. Comput. Chem. 34, 1429 (2013).

${ }^{304}$ S. F. Boys and F. Bernardi, Mol. Phys. 19, 553 (1970).

${ }^{305}$ S. M. Cybulski, G. Chalasiński, and R. Moszyński, J. Chem. Phys. 92, 4357 (1990).

${ }^{306}$ S. M. Cybulski and M. L. Lytle, J. Chem. Phys. 127, 141102 (2007).

${ }^{307}$ A. Heßelmann, J. Chem. Phys. 128, 144112 (2008).

${ }^{308}$ M. Pitonak and A. Heßelmann, J. Chem. Theory Comput. 6, 168 (2010).

${ }^{309}$ L. A. Burns, M. S. Marshall, and C. D. Sherrill, J. Chem. Phys. 141, 234111 (2014).

${ }^{310}$ B. Jeziorski, R. Moszynski, and K. Szalewicz, Chem. Rev. 94, 1887 (1994).

${ }^{311}$ K. Szalewicz, K. Patkowski, and B. Jeziorski, Struct.Bonding 116, 43 (2005).

${ }^{312}$ K. Szalewicz, Wiley Interdiscip. Rev.: Comput. Mol. Sci. 2, 254 (2012). 
${ }^{313}$ K. Patkowski, B. Jeziorski, T. Korona, and K. Szalewicz, J. Chem. Phys. 117, 5124 (2002).

${ }^{314}$ M. Jeziorska, B. Jeziorski, and J. Č́́žek, Int. J. Quantum Chem. 32, 149 (1987).

${ }^{315}$ A. Heßelmann and G. Jansen, Chem. Phys. Lett. 357, 464 (2002).

${ }^{316}$ A. Heßelmann and G. Jansen, Chem. Phys. Lett. 362, 319 (2002).

${ }^{317}$ A. Heßelmann and G. Jansen, Chem. Phys. Lett. 367, 778 (2003).

${ }^{318}$ A. Heßelmann and G. Jansen, Phys. Chem. Chem. Phys. 5, 5010 (2003).

${ }^{319}$ A. J. Misquitta and K. Szalewicz, Chem. Phys. Lett. 357, 301 (2002).

${ }^{320}$ A. Misquitta, B. Jeziorski, and K. Szalewicz, Phys. Rev. Lett. 91, 033201 (2003).

${ }^{321}$ A. J. Misquitta, R. Podeszwa, B. Jeziorski, and K. Szalewicz, J. Chem. Phys. 123, 214103 (2005)

${ }^{322}$ G. Jansen, Wiley Interdiscip. Rev.: Comput. Mol. Sci. 4, 127 (2013).

${ }^{323}$ R. Podeszwa, R. Bukowski, and K. Szalewicz, J. Phys. Chem. A 110, 10345 (2006).

${ }^{324} \mathrm{~A}$. Heßelmann, "The ring and exchange-ring approximations based on KohnSham reference states," in Density Functionals: Thermochemistry, Springer Series in Topics in Current Chemistry (Springer, Heidelberg, 2014).

${ }^{325}$ R. Podeszwa, J. Chem. Phys. 132, 044704 (2010).

${ }^{326} \mathrm{R}$. Schäffer and G. Jansen, Theor. Chem. Acc. 131, 1235 (2012).

${ }^{327}$ R. Schäffer and G. Jansen, Mol. Phys. 111, 2570 (2013).

${ }^{328}$ A. J. Misquitta, J. Chem. Theory Comput. 9, 5313 (2013).

${ }^{329}$ R.-F. Liu, C. A. Franzese, R. Malek, P. S. Żuchowski, J. G. Ángyán, M. M. Szczęśniak, and G. Chałasiński, J. Chem. Theory Comput. 7, 2399 (2011).

${ }^{330}$ T. Clark and A. Heßelmann, Phys. Chem. Chem. Phys. 20, 22849 (2018).

${ }^{331}$ A. Görling, Int. J. Quantum Chem. 69, 265 (1998).

${ }^{332}$ A. Heßelmann, J. Chem. Theory Comput. 14, 1943 (2018).

${ }^{333}$ S. Grimme, Chem. Eur. J. 18, 9955 (2012).

${ }^{334} \mathrm{~T}$. Risthaus and S. Grimme, J. Chem. Theory Comput. 9, 1580 (2013).

${ }^{335}$ T. Korona, A. Heßelmann, and H. Dodziuk, J. Chem. Theory Comput. 5, 1585 (2009).

${ }^{336}$ H. Dodziuk, K. Ruud, T. Korona, and T. B. Demissie, Phys. Chem. Chem. Phys. 18, 26057 (2016).

${ }^{337}$ T. Korona and B. Jeziorski, J. Chem. Phys. 125, 184109 (2006).

${ }^{338}$ T. Korona, Phys. Chem. Chem. Phys. 10, 6509 (2008).

${ }^{339}$ T. Korona, Phys. Chem. Chem. Phys. 10, 5698 (2008).

${ }^{340} \mathrm{~T}$. Korona and B. Jeziorski, J. Chem. Phys. 128, 144107 (2008).

${ }^{341}$ T. Korona, J. Chem. Theory Comput. 5, 2663 (2009).

${ }^{342}$ T. Korona, Mol. Phys. 111, 3705 (2013).

${ }^{343}$ T. H. Dunning, Jr., J. Chem. Phys. 90, 1007 (1989).

${ }^{344}$ R. Feng and K. A. Peterson, J. Chem. Phys. 147, 084108 (2017).

${ }^{345}$ J. G. Hill and K. A. Peterson, J. Chem. Phys. 147, 244106 (2017).

${ }^{346} \mathrm{~K}$. A. Peterson, in Computational Inorganic and Bioinorganic Chemistry, edited by E. I. Solomon, R. A. Scott, and R. B. King (Wiley \& Sons, Chichester, 2009), pp. $187-200$.
${ }^{347}$ J. G. Hill, A. Mitrushchenkov, K. E. Yousaf, and K. A. Peterson, J. Chem. Phys. 135, 144309 (2011).

${ }^{348}$ J. G. Hill and K. A. Peterson, Phys. Chem. Chem. Phys. 12, 10460 (2010).

${ }^{349}$ D. Feller and K. A. Peterson, J. Chem. Phys. 139, 084110 (2013).

${ }^{350}$ K. A. Peterson, M. K. Kesharwani, and J. M. L. Martin, Mol. Phys. 113, 1551 (2015).

${ }^{351}$ F. Weigend and R. Ahlrichs, Phys. Chem. Chem. Phys. 7, 3297 (2005).

${ }^{352}$ R. Gulde, P. Pollak, and F. Weigend, J. Chem. Theory Comput. 8, 4062 (2013).

${ }^{353}$ P.-O. Widmark, P.-Å. Malmqvist, and B. O. Roos, Theor. Chim. Acta 77, 291 (1990).

${ }^{354}$ B. O. Roos, R. Lindh, P.-Å. Malmqvist, V. Veryazov, P.-O. Widmark, and A. C. Borin, J. Phys. Chem. A 112, 11431 (2008).

${ }^{355}$ F. Weigend, J. Comput. Chem. 29, 167 (2008).

${ }^{356}$ F. Weigend, M. Häser, H. Patzelt, and R. Ahlrichs, Chem. Phys. Lett. 294, 143 (1998).

${ }^{357}$ C. Hättig, Phys. Chem. Chem. Phys. 7, 59 (2005).

${ }^{358}$ S. Kritikou and J. G. Hill, J. Chem. Theory Comput. 11, 5269 (2015).

${ }^{359}$ K. E. Yousaf and K. A. Peterson, J. Chem. Phys. 129, 184108 (2008).

${ }^{360}$ K. E. Yousaf and K. A. Peterson, Chem. Phys. Lett. 476, 303 (2009).

${ }^{361}$ R. A. Shaw and J. G. Hill, J. Chem. Theory Comput. 13, 1691 (2017).

${ }^{362}$ See http://www.pqs-chem.com/software.php for information about the PQSMol program; accessed 20 December 2019.

${ }^{363}$ J. Baker, K. Wolinski, M. Malagoli, D. Kinghorn, P. Wolinski, G. Magyarfalvi, S. Saebo, T. Janowski, and P. Pulay, J. Comput. Chem. 30, 317-335 (2009).

${ }^{364}$ J. Baker, T. Janowski, K. Wolinski, and P. Pulay, Wiley Interdiscip. Rev.: Comput. Mol. Sci. 2, 63-72 (2012).

${ }^{365} \mathrm{See}$ https://gitlab.com/molpro/sjef for an overview of SJEF and source code; accessed 10 January 2020.

${ }^{366}$ See http://www.molpro.net/schema/molpro-output for the XML schema used in Molpro output; accessed 20 December 2019.

${ }^{367}$ M. Sibaev, "Python simple job execution framework," https://molpro.gitlab.io/ pysjef/, 2020.

${ }^{368}$ M. Sibaev, "Pysjef extension for molpro," https://gitlab.com/molpro/ pysjef_molpro, 2020.

${ }^{369}$ T. Kluyver, B. Ragan-Kelley, F. Pérez, B. Granger, M. Bussonnier, J. Frederic, K. Kelley, J. Hamrick, J. Grout, S. Corlay, P. Ivanov, D. Avila, S. Abdalla, and C. Willing, in Positioning and Power in Academic Publishing: Players, Agents and Agendas, edited by F. Loizides and B. Schmidt (IOS Press, 2016), pp. 87-90.

${ }^{370}$ P. J. Knowles and N. C. Handy, Comput. Phys. Commun. 54, 75 (1989).

${ }^{371}$ See https://gitlab.com/molpro/fcidump for for the definition of the FCIdump file format and software that manipulates it; accessed 20 December 2019.

${ }^{372} \mathrm{See}$ https://gitlab.com/molpro/molpro-plugin for the source code of the molpro-plugin library; accessed 20 December 2019.

${ }^{373}$ G. H. Booth, S. D. Smart, and A. Alavi, Mol. Phys. 112, 1855 (2014); arXiv:1305.6981. 